\title{
Epidemiology of sleep disorders during COVID-19 pandemic: A systematic scoping review
}

Authors:

Samia Tasnim, MD, MPH ${ }^{1,2}$; Mariya Rahman, MD ${ }^{1,2}$; Priyanka Pawar, $\mathrm{MPH}^{3}$; Xinli Chi, $\mathrm{PhD}^{4}$;

Qian Yu, PhD ${ }^{4,5}$; Liye Zou, $\mathrm{PhD}^{4,5}$; Abida Sultana, $\mathrm{MBBS}^{2,6}$; E. Lisako J. McKyer, $\mathrm{PhD}^{1}$, Ping Ma, PhD ${ }^{1}$; Md Mahbub Hossain, MD, MPH ${ }^{1,2,6}$

Affiliations:

${ }^{1}$ School of Public Health, Texas A\&M University, College Station, TX 77843, USA.

${ }^{2}$ EviSyn Health, Khulna 09000, Bangladesh.

${ }^{3}$ Mamta Foundation, India.

${ }^{4}$ Center for Lifestyle and Mental Health, School of Psychology, Shenzhen University, Shenzhen, 518060, China.

${ }^{5}$ Exercise \& Mental Health Laboratory, School of Psychology, Shenzhen University, 518060, China.

${ }^{6}$ Nature Study Society of Bangladesh, Khulna 09000, Bangladesh.

Corresponding author: Samia Tasnim, MD, MPH; School of Public Health, Texas A\&M University, College Station, TX 77843, USA. Email: tasnim@tamu.edu

Conflicts of interest: None

Funding: No funding was received to conduct this review.

Acknowledgment: None 


\begin{abstract}
:
Background: A growing burden of mental health problems has become a global concern amid the coronavirus disease (COVID-19) pandemic. Sleep disorders are major mental health problems associated with increased psychosocial stressors; however, no research synthesis is available on the epidemiology of it. In this systematic scoping review, we aimed to assess the current evidence on the epidemiological burden, associated factors, and interventions from the existing literature.
\end{abstract}

Method: Seven major health databases and additional sources were searched to identify, evaluate, and synthesize empirical studies on the prevalence and correlates of sleep disorders and available interventions. The Joanna Briggs Institute Methodology for Scoping Review were used, and the findings were reported using the Preferred Reporting Items for Systematic Reviews and MetaAnalyses extension for Scoping Reviews (PRISMA-ScR) checklist.

Results: A total of 78 articles were retrieved, the prevalence of sleeping disorders ranged from $2.3 \%$ to $76.6 \%$. Age, sex, level of education, physical and mental health, COVID-19 related factors, occupation especially being health care workers ( $\mathrm{HCW}$ ) were the main associated factors. Only two intentions were identified to address the issue.

Conclusion: The finding of this review indicated a high burden of sleep disorder with limited interventions that necessitate informing policymakers and practitioners to facilitate future research and implementations.

Keywords: Sleep Disorders; Insomnia; Mental Health; COVID-19; Coronavirus; Systematic Review; Scoping Review 


\section{Brief summary:}

Current Knowledge/Study Rationale: Despite the paramount importance of sleep for the physical and mental wellbeing of individuals, sleep hygiene is often neglected which resulted in a high prevalence of sleep disorders across the globe. This condition is likely to worsen amid this pandemic. This is the first systematic scoping review of sleep disorders during the COVID-19 pandemic. Study Impact: The findings of our study suggest a high prevalence of sleep disorder and highlight a wide range of socio-demographic factors to identify population groups vulnerable to the adverse outcomes of sleep disorder with limited interventions. These pieces of evidence will guide clinicians to make informed choices for better management of patients and aid public health professionals to prevent sleep disorder epidemic concurring with the current pandemic. 


\section{Introduction:}

Novel coronavirus disease or COVID-19, an acute respiratory illness caused by a newly discovered SARS-CoV-2 virus emerged in December $2019^{1,2}$. Since then, it has rapidly surged to Europe, especially Italy and Spain, the USA, and progressed to become a global pandemic. In the last couple of months, it's reported to be spreading to the countries in other parts of Asia, Africa, and Latin America. Globally, by September 27th, 2020, a total of 32,730,945 cases of COVID-19 has been reported, including 991,224 deaths ${ }^{3}$. Of note, the World Health Organization declared it a global pandemic on March 11th, and after the declaration, most of the countries worldwide entered nationwide lockdown to prevent the spread of the infection ${ }^{4}$. While the clinical care practitioners and public health experts were focused on containing the spread of the virus, the COVID-19 pandemic and related quarantine measures have taken a heavy toll on people's mental health ${ }^{4,5}$. Historically quarantine has been related to anxiety, depression, panic, irritability, somatic disorder, and insomnia ${ }^{6-8}$. Moreover, a high level of stress and trauma-related disorders are byproducts of being isolated ${ }^{5,9,10}$. Moreover, factors like an extended period of isolation, fear of infection, uncertainty, disappointment, fatigue, stigma, inadequate data and information regarding the disease, insufficient supplies, and economic damage also negatively impact individuals' psychological wellbeing ${ }^{11}$. Psychosocial stressors like anxiety, stress, altered lifestyle with little to no social support, and fear may affect the pattern of sleep among individual often leading to sleep disorders ${ }^{12,13}$.

Sleep is an essential physiological activity in keeping up with physical and mental wellbeing and better life quality ${ }^{14,15}$. Breach in the normal sleep cycle can lead to insufficient sleep and prolonged alertness, hence increasing the event of insomnia, nightmares, daytime instability, and fatigue ${ }^{14}$. Recent studies have shown that sleep disorders impact up to 1 in 4 adults ${ }^{16}$. They are also found to be associated with a wide range of adverse health outcomes, for example, increased risk of obesity, diabetes, hypertension, cerebrovascular diseases, malignancy, musculoskeletal diseases, septicemia, and metabolic syndrome ${ }^{14,15,17}$. Further evaluations have found that 50-70 million adults in the United States have at least one sleep disorder ${ }^{16}$, while the prevalence of sleep disorders among the Australians and Netherlanders are $20-35 \%{ }^{18}$ and $27.3 \%{ }^{17}$ respectively. Potential risk factors of sleep disorders include severe stressful circumstances, depression, anxiety, trauma, low socioeconomic condition, urban living, increased use of technology, and social media ${ }^{14,16,17}$. 
A substantial amount of evidence suggests a high prevalence of various forms of sleep disorders in the global community. This situation is likely to worsen in the current circumstances with numerous psychological stressors. Many studies have reported a growing burden of a wide range of mental disorders, including sleep disorders ${ }^{19}$. However, to the best of our knowledge, there is a scarcity of concrete evidence on the magnitude of sleep disorders among individuals affected by this pandemic. As there is a growing concern on mental health problems during COVID-19 research synthesis may play a critical role in understanding the burden of those problems addressing the same ${ }^{6,19}$. This scoping review aims to address this knowledge gap through systematically evaluating the current evidence on the epidemiological burden of sleep disorders, associated factors, and interventions addressing the problems. The specific questions for this scoping review are listed below:

- What is the epidemiological burden of sleep disorders among different populations during COVID-19?

- What are the factors associated with sleep disorders during COVID-19?

- What are the available interventions for addressing sleep disorders amid COVID-19?

\section{Methods and materials}

This scoping review was conducted using the Joanna Briggs Institute (JBI) methodology for scoping reviews ${ }^{20}$. Moreover, the findings of this review are reported using the Preferred Reporting Items for Systematic Reviews and Meta-Analyses extension for Scoping Reviews (PRISMA-ScR) checklist $^{21}$. The protocol of this scoping review has been registered with The Open Science Framework $^{22}$.

\subsection{Search strategy}

We searched MEDLINE, Embase, Academic Search Ultimate, Cumulative Index to Nursing and Allied Health Literature (CINAHL), Web of Science, and APA PsycInfo databases using the keywords with Boolean operators "AND/OR" as mentioned in Table 1. The search was first conducted on May $13^{\text {th }}, 2020$ and updated on August 12 $2^{\text {th }}, 2020$. 
Table 1: Search terms for this scoping review

\begin{tabular}{|c|c|}
\hline Search terms & $\begin{array}{l}\text { Keywords (searched within titles, abstracts, and subject } \\
\text { headings fields) }\end{array}$ \\
\hline 1 & $\begin{array}{l}\text { "COVID-19" OR "2019-nCoV” OR "2019 coronavirus" OR } \\
\text { "Wuhan coronavirus" OR "Wuhan virus" OR "Wuhan } \\
\text { pneumonia" OR "2019 novel coronavirus" OR "novel } \\
\text { coronavirus" OR "SARS-CoV-2" }\end{array}$ \\
\hline 2 & 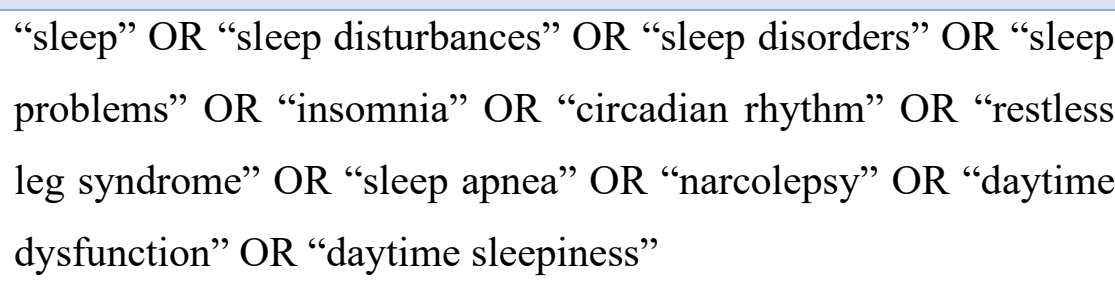 \\
\hline 3 & $\begin{array}{l}\text { prevalence OR incidence OR epidemiology OR frequency OR } \\
\text { case OR rate OR occurrence OR correlates OR determinants OR } \\
\text { predictors OR "risk factors" OR "associate factors" OR } \\
\text { interventions OR treatment OR therapy OR management }\end{array}$ \\
\hline Final search strategy & 1 AND 2 AND 3 \\
\hline
\end{tabular}

The keywords were searched within the titles, abstracts, and subject headings fields. Moreover, as COVID-19 literature started to get published since late 2019, we limited our search within 2019 and 2020. Moreover, we searched the reference lists and citing articles in Google Scholar to identify additional articles that could have met our criteria.

\subsection{Inclusion criteria}

\subsubsection{Participants}

In this scoping review, we included participants irrespective of their sociodemographic conditions. This makes our review inclusive for all types of participants who fulfill remaining criteria of this review.

\subsubsection{Concepts}


This review focused on sleep disorders, which can be defined by the International Classification of Diseases or Diagnostic and Statistical Manual of Mental Disorders ${ }^{23,24}$. Moreover, sleep abnormalities expressed as insomnia, excessive sleepiness, poor sleep quality, and abnormal events that occur during sleep will also be considered as sleep disorders in this review ${ }^{25}$. Studies reporting the prevalence, incidence, frequency, score, level or any forms of quantitative assessment of sleep-related conditions were included in this review.

\subsubsection{Context}

This review especially emphasized on COVID-19 as the context. Therefore, studies conducted among populations affected by COVID-19 (doctors or patients) or population at risk (general population who could have had infected with COVID-19) were be considered in this review. Moreover, studies without mentioning relevance to COVID-19 were excluded from this review.

\subsubsection{Types of sources}

This review included original studies, cross-sectional or longitudinal in nature, published as peerreviewed journal articles. Studies published in English language were included in this review. Therefore, unpublished works, non-original articles (for example, letters with no original research reports, editorials, reviews, commentaries etc.), non-peer reviewed articles, and studies in languages other than English were excluded from this review.

\subsubsection{Study selection}

After searching the databases, all the citations were imported to Rayyan QCRI, a cloud-based software for systematic reviews ${ }^{26}$. Two authors (ST and MR) independently evaluated those citations using the inclusion criteria of this review as stated earlier. At the end of independent screening, potential conflicts were reviewed and resolved based on discussion with a third author (MMH). Finally, the full texts of the included citations were assessed and articles meeting all the criteria were considered for data extraction. A flow chart of the study selection process is depicted in Figure 1. 
medRxiv preprint doi: https://doi.org/10.1101/2020.10.08.20209148; this version posted October 11, 2020. The copyright holder for this preprint (which was not certified by peer review) is the author/funder, who has granted medRxiv a license to display the preprint in perpetuity.

It is made available under a CC-BY 4.0 International license.

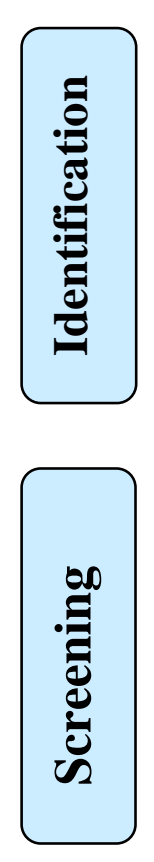

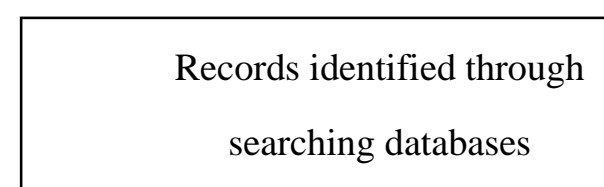

$(n=291)$
Additional records identified through other sources

$$
(\mathrm{n}=87)
$$
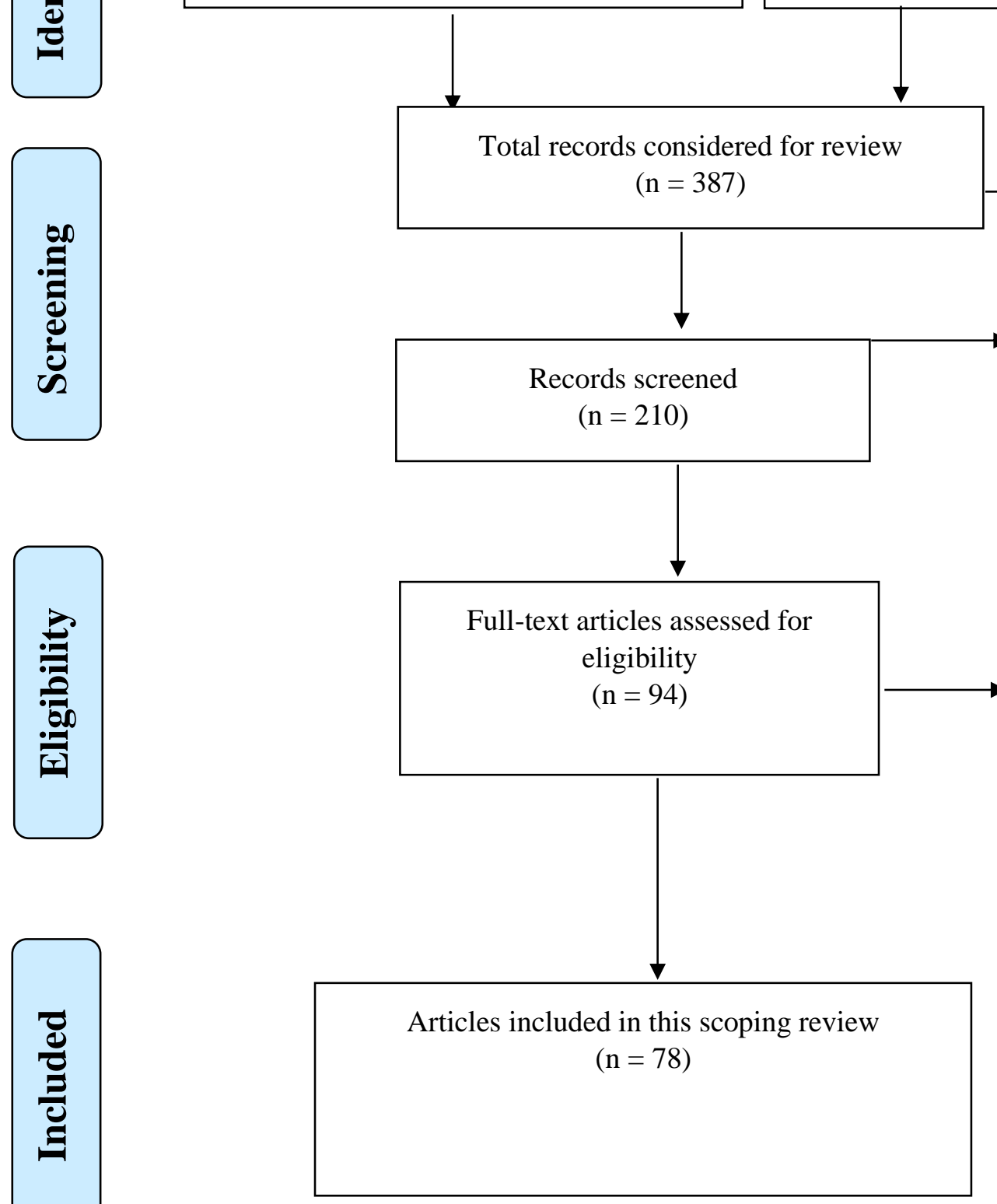

Records excluded $(\mathrm{n}=116)$ due to non-compliance with the inclusion or exclusion criteria

Full-text articles excluded $(\mathrm{n}=16)$ due to:

Study did not focus on any of the objectives of this review $(n=7)$

Sleep disorder was not assessed epidemiologically $(n=6)$

The study was not empirical in nature $(\mathrm{n}=3)$

Figure 1: Flow diagram of the systematic scoping review 


\subsection{Data extraction}

A data extraction form was prepared as shown in Table 2. Two authors (ST and MR) independently extracted data using this checklist. At the end of this phase, data for each article were reviewed by a third author to check consistency and potential conflicts were re-assessed by three reviewers and a consensus was made based on discussions. The finalized dataset was reviewed independently by two more co-authors (PP and AS) before final synthesis.

\subsection{Data presentation}

Data extracted from the included articles were narratively synthesized and presented using tables and a commentary on key findings on the study characteristics, samples, and epidemiological findings as the quantitative burden and associated factors of sleep disorders during COVID-19 and interventions addressing the same. As per the JBI methodology, scoping reviews do not aim to evaluate the quality of the studies. Therefore, no quality evaluation was done in this review. 
Table 2: Data extraction instrument

\begin{tabular}{|l|l|}
\hline Study information & Title \\
\hline & Author(s) \\
\hline & Year of publication \\
\hline & Country of origin \\
& Type of the study \\
\hline & Objective of the study \\
\hline Information on study & Sample size \\
\hline participants & \\
\hline
\end{tabular}

Sample characteristics

Recruitment strategy

Epidemiological data Methodology of the study

Timeframe of the study (with duration of exposure

or intervention, if available)

Prevalence or quantitative burden of sleep disorders

Factors (if reported) associated with sleep disorders

Interventions (if reported) addressing sleep disorders

(components and outcomes, if available)

Citation of the article Full citation of the article including digital object identifier (DOI)

References identified Articles that were cited in an article or forward from the cited articles citations of that article that may meet the criteria of this review 


\section{Result:}

We found 291 citations from searching the databases and 87 citations from additional sources, totaling 387 citations. After removing 177 duplicates, titles and abstracts of 210 articles were evaluated and 94 articles met the pre-set inclusion criteria. From the remaining articles 16 articles were removed after evaluating the full text. Finally, 78 articles met all criteria and were included in this scoping review.

\subsection{Characteristics of the included studies:}

\subsubsection{Study design:}

Most of the studies $82 \%(n=64)$ were of cross-sectional design. For example, Zhang et al., 2020 conducted cross-sectional survey among the hospital workers in China. A few included studies $(n=6)$ were of cohort studies. For example, the study by Nalleballe et al., $2020^{27}$ reported the findings from their cohort of patients of COVID-19. Remaining were case-control $(n=4)$ and pre-post study $(\mathrm{n}=3)$ design.

\subsubsection{Setting:}

Almost half of the studies $(\mathrm{n}=36,46 \%)$ were based on community setting. For example, the study by Gao et al., ${ }^{28}$ recruited 699 American adult participants to find out the effects of the pandemic related lockdown on their mental health. However, other half of the studies $(\mathrm{n}=37$, $47 \%$ ) recruited participants from hospitals or clinics. The study by Amerio et al., ${ }^{29}$ included general practitioners working in Genoa, Italy evaluate the mental health effects of the Covid-19 pandemic on the healthcare workers.

\subsubsection{Geographical scope:}

One third of the studies, $(\mathrm{n}=25,32 \%)$ were conducted in urban areas. For example, $76.5 \%$ of the participants recruited in the study by Voitsidis et al., ${ }^{30}$ were urban citizens. Similarly, the study by Xiao, Zhang, Kong, Li, \& Yang et al., ${ }^{31}$ reported $89.4 \%$ participants were recruited from urban area. In $13.75 \%(n=11)$ of the studies participants lived equally in urban and rural areas. For example, Renzo et al., ${ }^{32}$ reported that the percentage of participants living in rural and urban areas were comparable. However, in a few studies $(\mathrm{n}=18,23 \%)$ participants also came from small towns or rural areas. 


\subsubsection{Country:}

47.5\% ( $\mathrm{n}=38)$ of the studies were conducted in China. The study by Fu et al., ${ }^{33}$ was based on Chinese population where they recruited participants from Wuhan, China. $8.75 \%(\mathrm{n}=7)$ of the studies were based on USA. Wright et al., ${ }^{34}$ was an observational study based on the university students of the USA. $8.75 \%(n=7)$ of the studies were based on Italy. For example, the study by Gualano et al., ${ }^{35}$ was based on Italy.

\subsubsection{Sample:}

A large variation was noted in the sample size of the included studies. The sample size ranged from 26 to 40,469. Studies that provided intervention or conducted in-person assessments had a significantly smaller sample size. For example, Liu et al., ${ }^{36}$ conducted a randomized control trial of progressive muscle relaxation for insomnia among the COVID-19 patients in a hospital setting and had a small sample size of 51 participants. Similarly, Korkmaz et al., ${ }^{37}$ conducted face to face evaluation of several mental health conditions of health care workers employed in service for COVID-19 and had a sample size of 140 participant only.

However, studies that utilized the online surveys to collect data had a relatively larger sample size. For example, S. J. Zhou et al., ${ }^{38}$ collected data from 11,835 participants in China via online survey forms.

\subsubsection{Sampling technique:}

$65 \%$ of the studies $(n=51)$ mentioned specific sampling techniques. Among them, $35 \%$ of the studies $(\mathrm{n}=27)$ used the convenient sampling technique to recruit the participants. For example, Diomidous,et al., ${ }^{39}$ used convenient sampling technique to include health care providers for their study. Some studies $(n=6)$ also used the random sampling technique. For example, Liu et al., ${ }^{36}$ randomly selected 51 patients from the list of patients with confirmed COVID-19 admitted to the Hainan General Hospital for participating in their study. A few studies used clustered sampling technique. For example, Abdulah and colleagues' 40 used the clustered sampling technique. They obtained a list of local physicians who work in different medical settings. The participants from one pediatric, one emergency, one special corona, and one maternity and gynecology hospital were invited to participate in their online survey. 


\subsubsection{Disorders investigated:}

All the studies assessed insomnia/sleep disorder or quality of sleep. Most of the studies (n $=38,49 \%$ ) also investigated the level of anxiety among participants. For example, Mazza et al., ${ }^{41}$ assessed prevalence of anxiety among the COVID-19 survivors in Italy. Depression/depressive symptoms were assessed by 88 studies. For example, Huang et al., ${ }^{42}$ measured the burden of depression among Chinese public during the outbreak. Loneliness, suicidal ideation, somatic disorders, and Obsessive-Compulsive Disorder (OCD) were among other less frequently assessed disorders.

\subsubsection{Mode of data collection:}

$85 \%$ of the studies $(n=66)$ collected data through online surveys. For example, W.D.S. et al., ${ }^{43}$ collected data using online surveys on different social media platforms. Similarly, J et al., ${ }^{44}$ collected data from the Chinese population using the WeChat (the most widely used mobile app). Only $7 \%$ of the studies utilized more than one means of data collection. For example, Renzo et al., ${ }^{32}$ collected data using face to face interview, online survey and from reviewing hospital records of the patients. Remaining studies $(n=6,7 \%)$ collected data solely from face to face interviews. For example, Türkoğlu et al., ${ }^{45}$ collected data from in person evaluation of the children who participated in the study.

\subsubsection{Screening instruments and cut off value:}

Majority of the studies ( $\mathrm{n}=50,64 \%)$ employed the PSQI for diagnosing the severity of the insomnia. For example, Huang \& Zhao et al., ${ }^{42}$ used the Chinese version of PSQI whereas, the study by Innocenti et al., ${ }^{46}$ used the Italian version of PSQI among their participant. A little over half of the studies $(n=42,53 \%)$ used the ISI scale for diagnosing insomnia. Gualano et al., ${ }^{35}$ used ISI scale to diagnose insomnia among the Italian population. Few studies $(\mathrm{n}=15,19 \%)$ also utilized the AIS scale for diagnosis. For example, Tselebis et al., ${ }^{47}$ used AIS for diagnosing insomnia among COVID-19 survivors.

Majority of the studies used score of $\leq 7$ for PSQI, $\leq 10$ for ISI, and $\leq 6$ for AIS scale as cut of value for diagnosing the severity of insomnia. 


\begin{tabular}{|c|c|c|c|c|c|c|c|c|c|c|c|c|c|c|}
\hline $\begin{array}{l}\text { Author } \\
\text {, Year }\end{array}$ & $\begin{array}{l}\text { Study } \\
\text { design }\end{array}$ & Setting & $\begin{array}{l}\text { Level } \\
\text { of } \\
\text { educa } \\
\text { tion }\end{array}$ & Country & $\begin{array}{l}\text { Popula } \\
\text { tion } \\
\text { specifi } \\
\text { cs } \\
\end{array}$ & $\begin{array}{l}\text { Mean } \\
\text { age } \\
\text { (year } \\
\text { s) }\end{array}$ & $\begin{array}{l}\text { Disorder } \\
\text { investigat } \\
\text { ed }\end{array}$ & $\begin{array}{l}\text { Mode } \\
\text { of } \\
\text { interv } \\
\text { iew } \\
\end{array}$ & $\begin{array}{l}\text { Screening } \\
\text { instrume } \\
\text { nt }\end{array}$ & $\begin{array}{l}\text { Cut } \\
\text { off } \\
\text { valu } \\
\text { e } \\
\end{array}$ & $\begin{array}{l}\text { Samp } \\
\text { le size }\end{array}$ & $\begin{array}{l}\% \text { of } \\
\text { males } \\
\text { in the } \\
\text { sample }\end{array}$ & $\begin{array}{l}\text { Sampling } \\
\text { technique }\end{array}$ & Key findings \\
\hline $\begin{array}{l}\text { Abdull } \\
\text { ah et } \\
\text { al., } \\
2020\end{array}$ & $\begin{array}{l}\text { Cross- } \\
\text { section } \\
\text { al }\end{array}$ & $\begin{array}{l}\text { Hospita } \\
1 / \text { medic } \\
\text { al } \\
\text { setting }\end{array}$ & $\begin{array}{l}\text { Gradu } \\
\text { ate/ } \\
\text { Speci } \\
\text { alized }\end{array}$ & Iraq & $\begin{array}{l}\text { Healthc } \\
\text { are } \\
\text { provide } \\
\text { rs }\end{array}$ & $\begin{array}{l}35.06 \\
\pm 7.61\end{array}$ & $\begin{array}{l}\text { Insomnia } \\
\text { and } \\
\text { Anxiety }\end{array}$ & $\begin{array}{l}\text { Onlin } \\
\mathrm{e}\end{array}$ & AIS & 6 & 400 & $70.10 \%$ & $\begin{array}{l}\text { Clustered } \\
\text { sampling }\end{array}$ & $\begin{array}{l}\text { The mean sleep score of physicians } \\
\text { were } 8.43 / 24 \text {. More than two-thirds } \\
(68.3 \%) \text { of the physicians were } \\
\text { sleepless. Dealing with suspected or } \\
\text { confirmed cases of COVID- } 19 \text {, } \\
\text { increased stress and increase in number } \\
\text { of days dealing with confirmed COVID } \\
\text { cases had negative effect on sleep of } \\
\text { the physicians. }\end{array}$ \\
\hline $\begin{array}{l}\text { Anzar } \\
\text { et al., } \\
2020\end{array}$ & $\begin{array}{l}\text { Cross- } \\
\text { section } \\
\text { al }\end{array}$ & $\begin{array}{l}\text { Comm } \\
\text { unity }\end{array}$ & & Pakistan & $\begin{array}{l}\text { Genera } \\
1 \\
\text { populat } \\
\text { ion }\end{array}$ & $\begin{array}{l}25 \pm \\
0.4\end{array}$ & $\begin{array}{l}\text { Anxiety, } \\
\text { Insomnia, } \\
\text { Depressio } \\
\mathrm{n}\end{array}$ & $\begin{array}{l}\text { Onlin } \\
\mathrm{e}\end{array}$ & SPSQI & $\begin{array}{l}>18 \\
\text { in } \\
\text { scale }\end{array}$ & 303 & $32 \%$ & $\begin{array}{l}\text { Not } \\
\text { specified }\end{array}$ & $\begin{array}{l}\text { Prevalence of sleep disturbance was } \\
6.9 \% \text {, Quality of sleep was reduced } \\
\text { among males and health care workers }\end{array}$ \\
\hline $\begin{array}{l}\text { Batool- } \\
\text { Anwat } \\
\text { et., } \\
2020\end{array}$ & $\begin{array}{l}\text { Retrosp } \\
\text { ective } \\
\text { cohort }\end{array}$ & $\begin{array}{l}\text { Hospita } \\
1 / \text { medic } \\
\text { al } \\
\text { setting }\end{array}$ & & USA & $\begin{array}{l}\text { Patient } \\
\text { s of } \\
\text { Obstru } \\
\text { ctive } \\
\text { sleep } \\
\text { apnea }\end{array}$ & $\begin{array}{l}63.5 \pm \\
13.9\end{array}$ & $\begin{array}{l}\text { Insomnia, } \\
\text { Anxiety }\end{array}$ & $\begin{array}{l}\text { Onlin } \\
\text { e and } \\
\text { Recor } \\
\text { d } \\
\text { revie } \\
\text { w } \\
\end{array}$ & & & 123 & $55 \%$ & $\begin{array}{l}\text { Not } \\
\text { specified }\end{array}$ & $\begin{array}{l}\text { After the lockdown, there was an } \\
\text { increase in the number of patients with } \\
\text { insomnia ( } 41 \text { vs. } 48 \%, p=0.02 \text { ). Women } \\
\text { had higher prevalence of insomnia }\end{array}$ \\
\hline $\begin{array}{l}\text { Beck et } \\
\text { al., } \\
2020\end{array}$ & $\begin{array}{l}\text { Cross- } \\
\text { section } \\
\text { al }\end{array}$ & $\begin{array}{l}\text { Comm } \\
\text { unity }\end{array}$ & & France & $\begin{array}{l}\text { Mix of } \\
\text { COVI } \\
\text { D } \\
\text { positiv } \\
\text { e, } \\
\text { suspect } \\
\text { ed and } \\
\text { healthy }\end{array}$ & $\begin{array}{l}26 \% \\
<35\end{array}$ & Insomnia & $\begin{array}{l}\text { Onlin } \\
\mathrm{e}\end{array}$ & $\begin{array}{l}\text { Self- } \\
\text { designed }\end{array}$ & & 1,005 & & $\begin{array}{l}\text { Random } \\
\text { stratified } \\
\text { sampling }\end{array}$ & $\begin{array}{l}74 \% \text { of the participants reported trouble } \\
\text { sleeping compared with a prevalence } \\
\text { rate of } 49 \% \text { in the last general } \\
\text { population survey. Women reported } \\
\text { more sleeping problems than men, with } \\
\text { greater frequency or severity: } 31 \% \text { vs. } \\
16 \% \text { (1,005 subjects) reported trouble } \\
\text { sleeping compared with a prevalence } \\
\text { rate of } 49 \% \text { in the last general } \\
\text { population survey. Younger population } \\
\text { reported higher sleep disturbance } \\
\text { compared to older population ( } 79 \% \text { vs } \\
72 \%) \text { and their sleep disturbance started } \\
\text { after the lockdown and instate of } \\
\text { sleeping pills increased after lockdown } \\
(41 \% \text { vs } 16 \% \text { ). }\end{array}$ \\
\hline $\begin{array}{l}\text { Bharga } \\
\text { va et } \\
\text { al., } \\
2020\end{array}$ & $\begin{array}{l}\text { Cross- } \\
\text { section } \\
\text { al }\end{array}$ & $\begin{array}{l}\text { Hospita } \\
1 / \text { medic } \\
\text { al } \\
\text { setting }\end{array}$ & $\begin{array}{l}\text { Post } \\
\text { gradu } \\
\text { ate }\end{array}$ & USA & $\begin{array}{l}\text { Board- } \\
\text { certifie } \\
\text { d } \\
\text { dermat } \\
\text { ologists }\end{array}$ & & $\begin{array}{l}\text { Insomnia, } \\
\text { Stress, } \\
\text { Depressio } \\
\text { n, Mental } \\
\text { distress }\end{array}$ & $\begin{array}{l}\text { Onlin } \\
\mathrm{e}\end{array}$ & $\begin{array}{l}\text { Self- } \\
\text { designed }\end{array}$ & & 733 & & $\begin{array}{l}\text { Convenie } \\
\text { nt } \\
\text { sampling }\end{array}$ & $\begin{array}{l}30 \% \text { of the responders reported of } \\
\text { insomnia, }\end{array}$ \\
\hline $\begin{array}{l}\text { Zhang } \\
\text { et al., } \\
2020\end{array}$ & $\begin{array}{l}\text { Cross- } \\
\text { section } \\
\text { al }\end{array}$ & $\begin{array}{l}\text { Hospita } \\
1 / \text { medic } \\
\text { al } \\
\text { setting }\end{array}$ & $\begin{array}{l}\text { Gradu } \\
\text { ate }\end{array}$ & China & $\begin{array}{l}\text { Hospita } \\
1 \\
\text { worker } \\
\mathrm{s}\end{array}$ & $\begin{array}{l}\text { Not } \\
\text { report } \\
\text { ed }\end{array}$ & Insomnia & $\begin{array}{l}\text { Onlin } \\
\mathrm{e}\end{array}$ & ISI & $\geq 8$ & 1563 & $15 \%$ & $\begin{array}{l}\text { Random } \\
\text { sampling }\end{array}$ & $\begin{array}{l}36.1 \% \text { reported insomnia. They found } \\
\text { education level of high school or below } \\
(\mathrm{OR}=2.69) \text {, being a doctor }(\mathrm{OR}= \\
0.44) \text {,currently working in an isolation } \\
\text { unit }(\mathrm{OR}=1.71) \text {, worry about being } \\
\text { infected }(\mathrm{OR}=2.30, \text {, perceived lack of }\end{array}$ \\
\hline
\end{tabular}




\begin{tabular}{|c|c|c|c|c|c|c|c|c|c|c|c|c|c|c|}
\hline & & & & & & & & & & & & & & $\begin{array}{l}\text { helpfulness in terms of psychological } \\
\text { support from news or social media with } \\
\text { regard to } \\
\text { COVID-19 }(\mathrm{OR}=2.10) \text {, and having } \\
\text { very strong uncertainty regarding } \\
\text { effective disease control }(\mathrm{OR}=3.30) \\
\text { were associated with insomnia. }\end{array}$ \\
\hline $\begin{array}{l}\text { Carriga } \\
\text { n et al., } \\
2020\end{array}$ & $\begin{array}{l}\text { Cross- } \\
\text { section } \\
\text { al }\end{array}$ & $\begin{array}{l}\text { Comm } \\
\text { unity }\end{array}$ & $\begin{array}{l}\text { Gradu } \\
\text { ate }\end{array}$ & UK & $\begin{array}{l}\text { Individ } \\
\text { uals } \\
\text { with } \\
\text { dement } \\
\text { ia or } \\
\text { care } \\
\text { about } \\
\text { the } \\
\text { disease }\end{array}$ & $\begin{array}{l}59.2 \pm \\
13.3\end{array}$ & Insomnia & $\begin{array}{l}\text { Onlin } \\
\mathrm{e}\end{array}$ & PSQI & $\geq 5$ & 3,474 & $25 \%$ & $\begin{array}{l}\text { Clustered } \\
\text { sampling }\end{array}$ & $\begin{array}{l}44.8 \% \text { reported of worsened sleep after } \\
\text { the lockdown. Low mood, anxiety and } \\
\text { suspected, proven or at risk of COVID- } \\
19 \text { symptoms were significantly } \\
\text { associated with worse sleep. Older } \\
\text { people's sleep quality was less affected } \\
\text { than younger people by COVID } \\
\text { lockdown }(\mathrm{p}<0.001) \text {. Better sleep } \\
\text { quality was associated with going } \\
\text { outside and exercising earlier, rather } \\
\text { than later. }\end{array}$ \\
\hline $\begin{array}{l}\text { Casagr } \\
\text { ande et } \\
\text { al., } \\
2020\end{array}$ & $\begin{array}{l}\text { Cross- } \\
\text { section } \\
\text { al }\end{array}$ & $\begin{array}{l}\text { Comm } \\
\text { unity }\end{array}$ & $\begin{array}{l}50 \% \\
\text { high } \\
\text { schoo } \\
1\end{array}$ & Italy & $\begin{array}{l}\text { Genera } \\
1 \\
\text { populat } \\
\text { ion }\end{array}$ & $\begin{array}{l}30.0 \pm \\
11.5\end{array}$ & $\begin{array}{l}\text { Insomnia, } \\
\text { Anxiety, } \\
\text { General } \\
\text { wellbeing }\end{array}$ & $\begin{array}{l}\text { Onlin } \\
\mathrm{e}\end{array}$ & PSQI & $\geq 5$ & 2291 & $25.30 \%$ & $\begin{array}{l}\text { Convenie } \\
\text { nt } \\
\text { sampling }\end{array}$ & $\begin{array}{l}57.1 \% \text { of participants reported poor } \\
\text { sleep quality. Youth and women, those } \\
\text { uncertain regarding } \\
\text { possible COVID-19 infection, and } \\
\text { greater fear of direct contact with those } \\
\text { infected by } \\
\text { COVID-19 had an increased risk of } \\
\text { developing sleep disturbances. Sleep } \\
\text { quality was related to GAD, PTSD } \\
\text { related to COVID-19 symptom }\end{array}$ \\
\hline $\begin{array}{l}\text { Dai., } \\
2020\end{array}$ & $\begin{array}{l}\text { Cross- } \\
\text { section } \\
\text { al }\end{array}$ & $\begin{array}{l}\text { Comm } \\
\text { unity }\end{array}$ & $\begin{array}{l}\text { Bache } \\
\text { lor }\end{array}$ & Malaysia & $\begin{array}{l}\text { Genera } \\
1 \\
\text { populat } \\
\text { ion }\end{array}$ & $\begin{array}{l}30 \\
\text { years }\end{array}$ & $\begin{array}{l}\text { Insomnia, } \\
\text { Anxiety, } \\
\text { Depressio } \\
\text { n, Distress }\end{array}$ & $\begin{array}{l}\text { Onlin } \\
\mathrm{e}\end{array}$ & AIS & $>5$ & 669 & $48 \%$ & $\begin{array}{l}\text { Stratified } \\
\text { sampling } \\
\text { based on } \\
\text { location } \\
\text { and } \\
\text { cluster } \\
\text { sampling } \\
\text { from } \\
\text { ethnic and } \\
\text { religious } \\
\text { groups }\end{array}$ & $\begin{array}{l}\text { Perceived health conditions, perceived } \\
\text { testing availability and age was } \\
\text { predictor of insomnia. }\end{array}$ \\
\hline $\begin{array}{l}\text { Diomid } \\
\text { ous'202 } \\
0\end{array}$ & $\begin{array}{l}\text { Cross- } \\
\text { section } \\
\text { al }\end{array}$ & $\begin{array}{l}\text { Hospita } \\
1 / \text { medic } \\
\text { al } \\
\text { setting }\end{array}$ & $\begin{array}{l}\text { Gradu } \\
\text { ate/Sp } \\
\text { eciali } \\
\text { zed }\end{array}$ & Greece & $\begin{array}{l}\text { Healthc } \\
\text { are } \\
\text { provide } \\
\text { rs }\end{array}$ & $\begin{array}{l}\text { Not } \\
\text { report } \\
\text { ed }\end{array}$ & Insomnia & $\begin{array}{l}\text { Onlin } \\
\mathrm{e}\end{array}$ & $\begin{array}{l}\text { Jenkins } \\
\text { sleep } \\
\text { Scale, the } \\
\text { Athens } \\
\text { Insomnia } \\
\text { Scale and } \\
\text { the } \\
\text { Sleep_50 } \\
\text { Questionn } \\
\text { aire. }\end{array}$ & & 204 & $\begin{array}{l}\text { Not } \\
\text { reporte } \\
\text { d }\end{array}$ & $\begin{array}{l}\text { Convenie } \\
\text { nt } \\
\text { sampling }\end{array}$ & $\begin{array}{l}37.1 \% \text { reported moderate and } 3 \% \\
\text { reported severe insomnia. Waking up in } \\
\text { the middle of the night and inadequate } \\
\text { duration of sleeping was common } \\
\text { complains. Lack of physical activity } \\
\text { was associate with sleep disorders }\end{array}$ \\
\hline
\end{tabular}




\begin{tabular}{|c|c|c|c|c|c|c|c|c|c|c|c|c|c|c|}
\hline $\begin{array}{l}\text { Fu' et } \\
\text { al., } \\
2020\end{array}$ & $\begin{array}{l}\text { Cross- } \\
\text { section } \\
\text { al }\end{array}$ & $\begin{array}{l}\text { Comm } \\
\text { unity }\end{array}$ & $\begin{array}{l}\text { Bache } \\
\text { lor }\end{array}$ & $\begin{array}{l}\text { Wuhan, } \\
\text { China }\end{array}$ & $\begin{array}{l}\text { Genera } \\
1 \\
\text { populat } \\
\text { ion }\end{array}$ & & $\begin{array}{l}\text { Anxiety, } \\
\text { depressio } \\
\mathrm{n}, \\
\text { Insomnia, } \\
\text { and } \\
\text { passive } \\
\text { coping }\end{array}$ & $\begin{array}{l}\text { Onlin } \\
\mathrm{e}\end{array}$ & AIS & $>5$ & 1242 & $30 \%$ & $\begin{array}{l}\text { Convenie } \\
\text { nt } \\
\text { sampling }\end{array}$ & $\begin{array}{l}30.0 \% \text { had a sleep disorder. Being } \\
\text { female }(\mathrm{OR}=1.62) \text {, having a monthly } \\
\text { income between } \\
1000 \text { and } 5000 \mathrm{CNY}(\mathrm{OR}=2.61) \text {, not } \\
\text { exercising }(\mathrm{OR}=1.85) \text {, having a higher } \\
\text { education level } \\
\text { (bachelor's degree and above) }(\mathrm{OR}= \\
1.40) \text { were the risk factors for sleep } \\
\text { disorder. }\end{array}$ \\
\hline $\begin{array}{l}\text { Gao et } \\
\text { al., } \\
2020\end{array}$ & $\begin{array}{l}\text { longitu } \\
\text { dinal, } \\
\text { cross- } \\
\text { section } \\
\text { al, and } \\
\text { retrosp } \\
\text { ective } \\
\text { recall }\end{array}$ & $\begin{array}{l}\text { Comm } \\
\text { unity }\end{array}$ & & USA & $\begin{array}{l}\text { Genera } \\
1 \\
\text { populat } \\
\text { ion }\end{array}$ & $\begin{array}{l}38.04 \\
\pm 11.6 \\
5\end{array}$ & Insomnia & $\begin{array}{l}\text { Onlin } \\
\mathrm{e}\end{array}$ & PSQI & $\geq 5$ & 699 & $55.22 \%$ & $\begin{array}{l}\text { Convenie } \\
\text { nt } \\
\text { sampling }\end{array}$ & $\begin{array}{l}\text { Average sleep quality was unchanged, } \\
\text { or even improved, earlier in the } \\
\text { pandemic. However, approximately } \\
25 \% \text { of participants reported that their } \\
\text { sleep quality had worsened, which was } \\
\text { explained by stress vulnerability, } \\
\text { caregiving, adverse life impact, shift } \\
\text { work, and presence of COVID-19 } \\
\text { symptoms. }\end{array}$ \\
\hline $\begin{array}{l}\text { Gaur et } \\
\text { al., } \\
2020\end{array}$ & $\begin{array}{l}\text { Cross- } \\
\text { section } \\
\text { al }\end{array}$ & $\begin{array}{l}\text { Comm } \\
\text { unity }\end{array}$ & $\begin{array}{l}\text { Gradu } \\
\text { ate }\end{array}$ & India & $\begin{array}{l}\text { Genera } \\
1 \\
\text { populat } \\
\text { ion } \\
\end{array}$ & & $\begin{array}{l}\text { Insomnia, } \\
\text { Anxiety, } \\
\text { General } \\
\text { wellbeing }\end{array}$ & $\begin{array}{l}\text { Onlin } \\
\mathrm{e}\end{array}$ & ISI & $\geq 10$ & 1015 & $64 \%$ & $\begin{array}{l}\text { Snowball } \\
\text { sampling }\end{array}$ & $\begin{array}{l}\text { Prevalence of depression was } 27 \% \text {. } \\
\text { Living in urban areas, living alone and } \\
\text { concern about livelihood were risk } \\
\text { factors of insomnia. }\end{array}$ \\
\hline $\begin{array}{l}\text { Gualan } \\
\text { o et al., } \\
2020\end{array}$ & $\begin{array}{l}\text { Cross- } \\
\text { section } \\
\text { al }\end{array}$ & $\begin{array}{l}\text { Comm } \\
\text { unity }\end{array}$ & $\begin{array}{l}\text { Bache } \\
\text { lor }\end{array}$ & Italy & $\begin{array}{l}\text { Genera } \\
1 \\
\text { populat } \\
\text { ion }\end{array}$ & $\begin{array}{l}\text { media } \\
\mathrm{n} \text { age } \\
\text { was } \\
42 \\
\text { years } \\
(\mathrm{IQR} \\
=23)\end{array}$ & $\begin{array}{l}\text { Insomnia, } \\
\text { depressio } \\
\text { n, anxiety }\end{array}$ & $\begin{array}{l}\text { Onlin } \\
\mathrm{e}\end{array}$ & ISI & $\geq 10$ & 1515 & 34.4 & $\begin{array}{l}\text { Convenie } \\
\text { nt } \\
\text { sampling }\end{array}$ & $\begin{array}{l}42.2 \% \text { reported of sleep disturbances } \\
\text { and, among them } 17.4 \% \text { were } \\
\text { moderate/severe insomnia. . Increasing } \\
\text { age, an absence of work-related } \\
\text { troubles and being married or being a } \\
\text { cohabitant reduced such a probability. } \\
\text { Being females, spending an increased } \\
\text { time on internet and having chronic } \\
\text { conditions were associated with a } \\
\text { higher prevalence of sleep disturbances. }\end{array}$ \\
\hline $\begin{array}{l}\text { Guo et } \\
\text { al., } \\
2020\end{array}$ & $\begin{array}{l}\text { Cross- } \\
\text { section } \\
\text { al }\end{array}$ & $\begin{array}{l}\text { Comm } \\
\text { unity }\end{array}$ & $\begin{array}{l}\text { Bache } \\
\text { lor }\end{array}$ & China & $\begin{array}{l}\text { Genera } \\
1 \\
\text { populat } \\
\text { ion } \\
\end{array}$ & & $\begin{array}{l}\text { Depressio } \\
\text { n, PTSD, } \\
\text { Insomnia }\end{array}$ & $\begin{array}{l}\text { Onlin } \\
\mathrm{e}\end{array}$ & PSQI & 7 & 2441 & $41.40 \%$ & $\begin{array}{l}\text { Quota } \\
\text { sampling }\end{array}$ & $\begin{array}{l}\text { Exposure to COVID-19(OR=1.77), } \\
\text { media news exposure and perceived } \\
\text { negative income were associated with } \\
\text { higher mental health problems }\end{array}$ \\
\hline $\begin{array}{l}\text { Gupta } \\
\text { et al., } \\
2020\end{array}$ & $\begin{array}{l}\text { Cross- } \\
\text { section } \\
\text { al }\end{array}$ & $\begin{array}{l}\text { Comm } \\
\text { unity }\end{array}$ & $\begin{array}{l}\text { Gradu } \\
\text { ate }\end{array}$ & India & $\begin{array}{l}\text { Genera } \\
1 \\
\text { populat } \\
\text { ion }\end{array}$ & $\begin{array}{l}37.32 \\
\pm 13.0 \\
9\end{array}$ & Insomnia & $\begin{array}{l}\text { Onlin } \\
\mathrm{e}\end{array}$ & ISI & $>14$ & 958 & $58.80 \%$ & $\begin{array}{l}\text { Convenie } \\
\text { nt } \\
\text { sampling }\end{array}$ & $\begin{array}{l}10 \% \text { of the group met the criteria for } \\
\text { clinically significant insomnia } \\
\text { according to ISI, compared to the pre- } \\
\text { lockdown period, there was a shift to a } \\
\text { later bedtime and waking time, with a } \\
\text { reduction in night-time sleep and an } \\
\text { increase in day-time napping. These } \\
\text { effects mostly affected working } \\
\text { individuals except health professionals. } \\
\text { Sleep quality deteriorated across all } \\
\text { occupational groups. Depressive } \\
\text { symptoms were associated with } \\
\text { reduction in duration of sleep. }\end{array}$ \\
\hline
\end{tabular}




\begin{tabular}{|c|c|c|c|c|c|c|c|c|c|c|c|c|c|c|}
\hline $\begin{array}{l}\text { Wang } \\
\text { et al., } \\
2020\end{array}$ & $\begin{array}{l}\text { Cross- } \\
\text { section } \\
\text { al }\end{array}$ & $\begin{array}{l}\text { Hospita } \\
1\end{array}$ & $\begin{array}{l}\text { Gradu } \\
\text { ate/ } \\
\text { specia } \\
\text { lized }\end{array}$ & China & $\begin{array}{l}\text { Health } \\
\text { care } \\
\text { worker } \\
\mathrm{s}\end{array}$ & & $\begin{array}{l}\text { Insomnia, } \\
\text { depressio } \\
\text { n, anxiety }\end{array}$ & $\begin{array}{l}\text { Onlin } \\
\mathrm{e}\end{array}$ & & & 1045 & $14.20 \%$ & $\begin{array}{l}\text { Convenie } \\
\text { nt } \\
\text { sampling }\end{array}$ & $\begin{array}{l}\text { The high-risk (o exposure) group had } \\
\text { higher levels of clinical insomnia } \\
(13.5 \% \text { v. } 8.5 \%, p=0.011) \text { and then the } \\
\text { low-risk group. Additionally, work } \\
\text { experience negatively correlated with } \\
\text { insomnia symptoms }\end{array}$ \\
\hline $\begin{array}{l}\text { Hao et } \\
\text { al., } \\
2020\end{array}$ & $\begin{array}{l}\text { Case- } \\
\text { control } \\
\text { study }\end{array}$ & $\begin{array}{l}\text { Hospita } \\
1\end{array}$ & $\begin{array}{l}\text { Bache } \\
\text { lor }\end{array}$ & China & $\begin{array}{l}\text { Psychia } \\
\text { tric } \\
\text { patients }\end{array}$ & $\begin{array}{l}32.8 \pm \\
11.8\end{array}$ & $\begin{array}{l}\text { Depressio } \\
\text { n, } \\
\text { Anxiety, } \\
\text { Stress and } \\
\text { Insomnia }\end{array}$ & $\begin{array}{l}\text { Onlin } \\
\mathrm{e}\end{array}$ & ISI & & 185 & & $\begin{array}{l}\text { Psychiatri } \\
\text { c patients } \\
\text { from the } \\
\text { databases } \\
\text { of the } \\
\text { First } \\
\text { People's } \\
\text { Hospital } \\
\text { of } \\
\text { Chongqin } \\
\text { g Liang } \\
\text { Jiang New } \\
\text { Area, } \\
\text { China. }\end{array}$ & $\begin{array}{l}\text { ISI scores were higher in psychiatric } \\
\text { patients than healthy controls (p < } \\
0.001) \text {. More than one-quarter of } \\
\text { psychiatric patients suffered from } \\
\text { moderately severe to severe insomnia. } \\
\text { Respondents who reported no change, } \\
\text { poor or worse physical health status } \\
\text { and had a psychiatric illness were } \\
\text { significantly more likely to have higher } \\
\text { mean ISI scores. }\end{array}$ \\
\hline $\begin{array}{l}\text { Huang } \\
\text { et ., } \\
2020\end{array}$ & $\begin{array}{l}\text { Cross- } \\
\text { section } \\
\text { al }\end{array}$ & $\begin{array}{l}\text { Comm } \\
\text { unity }\end{array}$ & $\begin{array}{l}\text { Bache } \\
\text { lor }\end{array}$ & China & $\begin{array}{l}\text { Genera } \\
1 \\
\text { populat } \\
\text { ion }\end{array}$ & $\begin{array}{l}35.3 \pm \\
5.6\end{array}$ & $\begin{array}{l}\text { Depressio } \\
\mathrm{n} \text {, Anxiety } \\
\text { and } \\
\text { Insomnia }\end{array}$ & $\begin{array}{l}\text { Onlin } \\
\mathrm{e}\end{array}$ & CPSQI & 7 & 7,236 & $45.40 \%$ & $\begin{array}{l}\text { Participan } \\
\text { ts from } \\
\text { National } \\
\text { Internet } \\
\text { Survey on } \\
\text { Emotional } \\
\text { and } \\
\text { Mental } \\
\text { Health } \\
\text { (NISEMH } \\
\text { ), }\end{array}$ & $\begin{array}{l}\text { The overall prevalence of sleep quality } \\
\text { was } 18.2 \% \text {. Compared with other } \\
\text { occupational group, healthcare } \\
\text { workers were more likely to have poor } \\
\text { sleep quality. }\end{array}$ \\
\hline $\begin{array}{l}\text { Idrissi } \\
\text { et al., } \\
2020\end{array}$ & $\begin{array}{l}\text { Cross- } \\
\text { section } \\
\text { al }\end{array}$ & $\begin{array}{l}\text { Comm } \\
\text { unity }\end{array}$ & $\begin{array}{l}\text { Bache } \\
\text { lor }\end{array}$ & Morocco & $\begin{array}{l}\text { Genera } \\
1 \\
\text { populat } \\
\text { ion }\end{array}$ & $\begin{array}{l}35.9 \pm \\
12.5\end{array}$ & $\begin{array}{l}\text { Insomnia, } \\
\text { Depressio } \\
\text { n, Anxiety }\end{array}$ & $\begin{array}{l}\text { Onlin } \\
\mathrm{e}\end{array}$ & $\begin{array}{l}\text { DBAS-16, } \\
\text { AIS, ESS }\end{array}$ & $\begin{array}{l}\text { DB } \\
\text { AS } \\
\geq 4, \\
\text { AIS } \\
\geq 6 \\
\text { ESS } \\
\geq 11\end{array}$ & 827 & $48.80 \%$ & $\begin{array}{l}\text { Convenie } \\
\text { nt } \\
\text { sampling }\end{array}$ & $\begin{array}{l}56 \% \text { reported insomnia, } 9.9 \% \text { reported } \\
\text { daytime sleepiness. Insomnia score was } \\
\text { higher in urban population }(\mathrm{OR}=2.09) \text {, } \\
\text { people with chronic disease }(\mathrm{OR}=2.14)\end{array}$ \\
\hline $\begin{array}{l}\text { Innoce } \\
\text { nti et } \\
\text { al., } \\
2020\end{array}$ & $\begin{array}{l}\text { Cross- } \\
\text { section } \\
\text { al }\end{array}$ & $\begin{array}{l}\text { Comm } \\
\text { unity }\end{array}$ & $\begin{array}{l}\text { Bache } \\
\text { lor }\end{array}$ & Italy & $\begin{array}{l}\text { Genera } \\
1 \\
\text { populat } \\
\text { ion }\end{array}$ & $\begin{array}{l}46.6 \% \\
\text { in the } \\
30-50 \\
\text { years } \\
\text { range }\end{array}$ & Insomnia & $\begin{array}{l}\text { Onlin } \\
\mathrm{e}\end{array}$ & IPSQI & 7 & 1035 & $17.10 \%$ & $\begin{array}{l}\text { Convenie } \\
\mathrm{nt} \\
\text { sampling }\end{array}$ & $\begin{array}{l}\text { Participants reported of } 1-2 \text {-hour delay } \\
\text { in awakening and sleeping time than } \\
\text { before the COVID-19 pandemic and to } \\
\text { take longer to fall asleep (those taking } \\
>1 \mathrm{~h} \text { to fall asleep increased from } 2.8 \\
\text { to } 16 \% \text { ). Nighttime awakenings also } \\
\text { were reported to be increased ( } 3 \text { out of } \\
4 \text { people woke up early at least once a } \\
\text { week vs. } 4 \text { out of } 10 \text { people before the } \\
\text { COVID- } 19 \text { pandemic) and increase in } \\
\text { taking sleeping pills was also noted. }\end{array}$ \\
\hline
\end{tabular}




\begin{tabular}{|c|c|c|c|c|c|c|c|c|c|c|c|c|c|c|}
\hline $\begin{array}{l}\text { Wang } \\
\text { et al., } \\
2020\end{array}$ & $\begin{array}{l}\text { Cross- } \\
\text { section } \\
\text { al }\end{array}$ & $\begin{array}{l}\text { Comm } \\
\text { unity }\end{array}$ & $\begin{array}{l}\text { Bache } \\
\text { lor }\end{array}$ & China & $\begin{array}{l}\text { Genera } \\
1 \\
\text { populat } \\
\text { ion }\end{array}$ & $\begin{array}{l}31.40 \\
\pm 13.4 \\
9\end{array}$ & Insomnia & $\begin{array}{l}\text { Onlin } \\
\mathrm{e}\end{array}$ & PSQI & 7 & 6437 & $43.87 \%$ & $\begin{array}{l}\text { Snowball } \\
\text { sampling }\end{array}$ & $\begin{array}{l}\text { Incidence of sleep disturbance among } \\
\text { residents was } 17.65 \% \text {. It was associated } \\
\text { with older age }(\mathrm{OR}=1.42) \text {, female } \\
\text { gender }(\mathrm{OR}=1.35) \text {, poor self-reported } \\
\text { health status }(\mathrm{OR}=5.59) \text {, who believed } \\
\text { COVID-19 was hard to cure }(\mathrm{OR}=1.73)\end{array}$ \\
\hline $\begin{array}{l}\text { Juliana } \\
\text { et al., } \\
2020\end{array}$ & $\begin{array}{l}\text { Pre- } \\
\text { post } \\
\text { study }\end{array}$ & $\begin{array}{l}\text { Comm } \\
\text { unity }\end{array}$ & & $\begin{array}{l}\text { Argentin } \\
\text { a }\end{array}$ & $\begin{array}{l}\text { Genera } \\
1 \\
\text { populat } \\
\text { ion }\end{array}$ & & & $\begin{array}{l}\text { Onlin } \\
\mathrm{e}\end{array}$ & PSQI & 7 & 1021 & & $\begin{array}{l}\text { Convenie } \\
\text { nt } \\
\text { sampling }\end{array}$ & $\begin{array}{l}\text { Sleep onset and offset were delayed } \\
\text { during the lockdown, weekday sleep } \\
\text { duration was longer. } 37.30 \% \text { of } \\
\text { participants did not reach the } \\
\text { recommended } 7 \text { h of sleep on weekdays } \\
\text { during the lockdown compared to } \\
60.24 \% \text { under control conditions. }\end{array}$ \\
\hline $\begin{array}{l}\text { Kokou- } \\
\text { Kpolou } \\
\text { et al., } \\
2020\end{array}$ & $\begin{array}{l}\text { Cross- } \\
\text { section } \\
\text { al }\end{array}$ & $\begin{array}{l}\text { Comm } \\
\text { unity }\end{array}$ & $\begin{array}{l}\text { Mixe } \\
\text { d }\end{array}$ & France & $\begin{array}{l}\text { Genera } \\
1 \\
\text { populat } \\
\text { ion }\end{array}$ & 30.06 & $\begin{array}{l}\text { Insomnia, } \\
\text { Lonelines } \\
\mathrm{s}\end{array}$ & $\begin{array}{l}\text { Onlin } \\
\mathrm{e}\end{array}$ & ISI & $>15$ & 556 & $25 \%$ & $\begin{array}{l}\text { Purposive } \\
\text { and } \\
\text { snowball } \\
\text { sampling }\end{array}$ & $\begin{array}{l}19.1 \% \text { met the diagnostic criteria of } \\
\text { clinical insomnia (ISI } \geq 15) \text { with the } \\
\text { mean total ISI scores of } 9.2(\mathrm{SD}=5.66) \\
\text { with. Individuals with postgraduate } \\
\text { levels had reported lower levels of } \\
\text { severity of insomnia than those with } \\
\text { college }(\mathrm{OR}=2.41,) \text { and undergraduate } \\
(\mathrm{OR}=2.59) \text { level education. Worries } \\
\text { about the COVID-19 }(\mathrm{OR}=1.39) \text {, fear } \\
\text { of being infected by the virus }(\mathrm{OR}= \\
0.43) \text {, pre-existing mental health illness } \\
(\mathrm{OR}=1.22) \text {, and loneliness }(\mathrm{OR}= \\
0.41) \text { increased the likelihood of being } \\
\text { diagnosed with clinical insomnia. }\end{array}$ \\
\hline $\begin{array}{l}\text { Li et } \\
\text { al., } \\
2020\end{array}$ & $\begin{array}{l}\text { Cross- } \\
\text { section } \\
\text { al }\end{array}$ & $\begin{array}{l}\text { Comm } \\
\text { unity }\end{array}$ & $\begin{array}{l}\text { Bache } \\
\text { lor }\end{array}$ & Taiwan & $\begin{array}{l}\text { Genera } \\
1 \\
\text { populat } \\
\text { ion }\end{array}$ & $\begin{array}{l}37.81 \\
\pm \\
11.00\end{array}$ & $\begin{array}{l}\text { Insomnia, } \\
\text { Suicidal } \\
\text { ideation }\end{array}$ & $\begin{array}{l}\text { Onlin } \\
\mathrm{e}\end{array}$ & $\begin{array}{l}\text { Self- } \\
\text { designed }\end{array}$ & & 1970 & $32.99 \%$ & $\begin{array}{l}\text { Convenie } \\
\text { nt } \\
\text { sampling }\end{array}$ & $\begin{array}{l}55.8 \% \text { of the participants reported sleep } \\
\text { disturbance, Increased worry about } \\
\text { COVID-19, more severe impact of } \\
\text { COVID-19 on social interaction, lower } \\
\text { perceived social support, more severe } \\
\text { academic/occupational interference due } \\
\text { to COVID-19, lower COVID-19- } \\
\text { specified support, and poorer self- } \\
\text { reported physical health were } \\
\text { significantly associated with sleep } \\
\text { disturbance. }\end{array}$ \\
\hline $\begin{array}{l}\text { Li et } \\
\text { al., } \\
2020\end{array}$ & $\begin{array}{l}\text { Case- } \\
\text { control } \\
\text { study }\end{array}$ & $\begin{array}{l}\text { Medica } \\
1 \text { staff }\end{array}$ & $\begin{array}{l}\text { Bache } \\
\text { lor }\end{array}$ & China & $\begin{array}{l}\text { Medica } \\
1 \text { staff } \\
\text { and } \\
\text { volunte } \\
\text { ers }\end{array}$ & $\begin{array}{l}20-40 \\
\text { years }\end{array}$ & Insomnia & $\begin{array}{l}\text { Onlin } \\
\mathrm{e}\end{array}$ & AIS & & & $13.18 \%$ & $\begin{array}{l}\text { Convenie } \\
\text { nt } \\
\text { sampling }\end{array}$ & $\begin{array}{l}\text { The medical staff in Wuhan had higher } \\
\text { insomnia than in Ningbo }(58.9 \% \text { vs. } \\
24.97 \% ; \mathrm{p}=0.001) \text {. The medical staff } \\
\text { in Wuhan, the symptoms of insomnia } \\
\text { were related to gender }(\mathrm{OR}=1.379) \text {, } \\
\text { education (OR }=1.54) \text {, and general } \\
\text { psychological symptoms }(\mathrm{OR}=2.124) \text {. } \\
\text { Among the medical staff in Ningbo, } \\
\text { insomnia was not only related to } \\
\text { general psychological symptoms (OR }= \\
1.60, \mathrm{P}<0.01,95 \% \mathrm{CI}=1.48-1.74) \text { but }\end{array}$ \\
\hline
\end{tabular}




\begin{tabular}{|c|c|c|c|c|c|c|c|c|c|c|c|c|c|c|}
\hline & & & & & & & & & & & & & & $\begin{array}{l}\text { also related to marital status }(\mathrm{OR}= \\
0.57)\end{array}$ \\
\hline $\begin{array}{l}\mathrm{Li} \text { et } \\
\text { al., } \\
2020\end{array}$ & $\begin{array}{l}\text { Cross- } \\
\text { section } \\
\text { al }\end{array}$ & $\begin{array}{l}\text { Comm } \\
\text { unity }\end{array}$ & & China & & $\begin{array}{l}34.46 \\
\pm 9.62\end{array}$ & $\begin{array}{l}\text { Insomnia, } \\
\text { Anxiety }\end{array}$ & $\begin{array}{l}\text { Onlin } \\
\mathrm{e}\end{array}$ & ISI & $>7$ & 3637 & $43 \%$ & $\begin{array}{l}\text { Convenie } \\
\text { nt } \\
\text { sampling }\end{array}$ & $\begin{array}{l}\text { The prevalence of insomnia increased } \\
\text { significantly (ISI > 7,26.2\% vs } 33.7 \% \text {, } \\
\mathrm{P}<. .001) ; 13.6 \% \text { developed new-onset } \\
\text { insomnia and } 12.5 \% \text { reported } \\
\text { worsened insomnia symptoms. Lengths } \\
\text { of time in bed ( } 485.5 \pm 72.6 \text { vs } 531.5 \\
\pm 94.2 \text { minutes) and total sleep time } \\
\text { ( } 432.8 \pm 65.6 \text { v } 466.9 \pm 95.6 \text { minutes) } \\
\text { increased significantly, and SE ( } 88.5 \% \\
\text { vs } 86.8 \% \text { ) decreased significantly. } \\
\text { Delayed bedtime ( } 25.6 \pm 66.3 \text { minutes) } \\
\text { and wakeup time ( } 71.7 \pm 89.5 \text { minutes) } \\
\text { were also observed }\end{array}$ \\
\hline $\begin{array}{l}\text { Li et } \\
\text { al., } \\
2020\end{array}$ & $\begin{array}{l}\text { Cross- } \\
\text { section } \\
\text { al }\end{array}$ & $\begin{array}{l}\text { Comm } \\
\text { unity }\end{array}$ & $\begin{array}{l}\text { Bache } \\
\text { lor }\end{array}$ & China & $\begin{array}{l}\text { Worker } \\
\text { with } \\
\text { income } \\
\text { loss }\end{array}$ & $26-40$ & $\begin{array}{l}\text { Depressio } \\
\text { n, anxiety, } \\
\text { insomnia, } \\
\text { and } \\
\text { distress }\end{array}$ & $\begin{array}{l}\text { Onlin } \\
\text { e and } \\
\text { Face } \\
\text { to } \\
\text { face }\end{array}$ & CISI & $>7$ & 398 & $50.50 \%$ & $\begin{array}{l}\text { Convenie } \\
\text { nt } \\
\text { sampling }\end{array}$ & $\begin{array}{l}\text { Prevalence on insomnia } 30.9 \% \text {. } \\
\text { Participants working in Hubei province } \\
\text { with heavy income losses, especially } \\
\text { pregnant women, were found to have a } \\
\text { high risk of developing had severe } \\
\text { insomnia }\end{array}$ \\
\hline $\begin{array}{l}\text { Lin et } \\
\text { al., } \\
2020\end{array}$ & $\begin{array}{l}\text { Cross- } \\
\text { section } \\
\text { al }\end{array}$ & $\begin{array}{l}\text { Comm } \\
\text { unity }\end{array}$ & $\begin{array}{l}\text { Bache } \\
\text { lor }\end{array}$ & China & $\begin{array}{l}\text { Genera } \\
1 \\
\text { populat } \\
\text { ion }\end{array}$ & $18-60$ & $\begin{array}{l}\text { Depressio } \\
\mathrm{n} \text {, anxiety, } \\
\text { insomnia, } \\
\text { and stress }\end{array}$ & $\begin{array}{l}\text { Onlin } \\
\mathrm{e}\end{array}$ & ISI & $>7$ & 5461 & $29.90 \%$ & $\begin{array}{l}\text { Convenie } \\
\text { nt } \\
\text { sampling }\end{array}$ & $\begin{array}{l}\text { Threat of COVID-19, age }<18, \text { male, } \\
\text { and living in Hubei province are } \\
\text { significantly correlated with insomnia } \\
(\mathrm{p}<0.01) \text { The prevalence of clinical } \\
\text { insomnia during the outbreak was } \\
20.05 \% \text { according to the ISI. } 20.01 \% \\
\text { participants spent more than one hour } \\
\text { awake in bed. }\end{array}$ \\
\hline $\begin{array}{l}\text { Macias } \\
\text { zeck et } \\
\text { al., } \\
2020\end{array}$ & $\begin{array}{l}\text { Cross- } \\
\text { section } \\
\text { al }\end{array}$ & $\begin{array}{l}\text { Comm } \\
\text { unity }\end{array}$ & $\begin{array}{l}\text { Gradu } \\
\text { ate }\end{array}$ & Poland & $\begin{array}{l}60 \% \\
\text { health } \\
\text { care } \\
\text { worker } \\
\text { s }\end{array}$ & $\begin{array}{l}39.23 \\
\pm \\
12.26\end{array}$ & $\begin{array}{l}\text { Anxiety, } \\
\text { insomnia, } \\
\text { somatic } \\
\text { symptoms }\end{array}$ & $\begin{array}{l}\text { Onlin } \\
\mathrm{e}\end{array}$ & & & 2039 & $20 \%$ & $\begin{array}{l}\text { Convenie } \\
\text { nt } \\
\text { sampling }\end{array}$ & $\begin{array}{l}\text { Insomnia are more prevalent among } \\
\text { medical staff than workers in other } \\
\text { professions. }\end{array}$ \\
\hline $\begin{array}{l}\text { Marelli } \\
\text { et al., } \\
2020\end{array}$ & $\begin{array}{l}\text { Cross- } \\
\text { section } \\
\text { al }\end{array}$ & $\begin{array}{l}\text { Comm } \\
\text { unity }\end{array}$ & $\begin{array}{l}\text { Highs } \\
\text { chool }\end{array}$ & Italy & $\begin{array}{l}\text { univers } \\
\text { ity } \\
\text { student } \\
\text { and } \\
\text { staff }\end{array}$ & $\begin{array}{l}22.84 \\
\pm 2.68\end{array}$ & $\begin{array}{l}\text { Insomnia, } \\
\text { Anxiety, } \\
\text { depressio } \\
\mathrm{n}\end{array}$ & $\begin{array}{l}\text { Onlin } \\
\mathrm{e}\end{array}$ & IPSQI & 7 & 400 & & $\begin{array}{l}\text { Convenie } \\
\text { nt } \\
\text { sampling }\end{array}$ & $\begin{array}{l}\text { Increase in bedtime hour, sleep } \\
\text { latency, and wake-up time between } \\
\text { before and during COVID- } 19 \\
\text { emergency and a worsening of sleep } \\
\text { quality and of insomnia symptoms. In } \\
\text { particular, during the lockdown, the } \\
\text { impact of the delay in bedtime and in } \\
\text { wake-up was more common among } \\
\text { students. In workers the prevalence if } \\
\text { insomnia was } 40 \% \text {, among them } 42 \% \\
\text { reported difficulty in sleep initiation. }\end{array}$ \\
\hline $\begin{array}{l}\text { Mazza } \\
\text { et al., } \\
2020\end{array}$ & $\begin{array}{l}\text { Cross- } \\
\text { section } \\
\text { al }\end{array}$ & $\begin{array}{l}\text { Hospita } \\
1\end{array}$ & & Italy & $\begin{array}{l}\text { COVI } \\
\text { D-19 } \\
\text { survivo } \\
r\end{array}$ & 57.8 & $\begin{array}{l}\text { Anxiety, } \\
\text { insomnia, } \\
\text { depressio } \\
\text { n, PTSD }\end{array}$ & $\begin{array}{l}\text { Face } \\
\text { to } \\
\text { face } \\
\text { and } \\
\text { online }\end{array}$ & $\begin{array}{l}\text { MOSSS, } \\
\text { WHIIRS }\end{array}$ & $>9$ & 402 & $65 \%$ & $\begin{array}{l}\text { Convenie } \\
\text { nt } \\
\text { sampling }\end{array}$ & $\begin{array}{l}\text { According to self-report } 40 \% \text { was } \\
\text { suffering from insomnia. Patients with } \\
\text { any psychiatric symptoms had a higher } \\
\text { immunological symptom }\end{array}$ \\
\hline
\end{tabular}




\begin{tabular}{|c|c|c|c|c|c|c|c|c|c|c|c|c|c|c|}
\hline $\begin{array}{l}\text { Pinto et } \\
\text { al., } \\
2020\end{array}$ & $\begin{array}{l}\text { Cross- } \\
\text { section } \\
\text { al }\end{array}$ & $\begin{array}{l}\text { Hospita } \\
1\end{array}$ & & Portugal & $\begin{array}{l}\text { Patient } \\
\text { s of } \\
\text { sleep } \\
\text { disorde } \\
\text { red } \\
\text { breathi } \\
\text { ng } \\
\end{array}$ & 63.9 & $\begin{array}{l}\text { Sleep } \\
\text { quality/ins } \\
\text { omnia }\end{array}$ & $\begin{array}{l}\text { Face } \\
\text { to } \\
\text { face } \\
\text { and } \\
\text { online }\end{array}$ & JSS & & 365 & $55.60 \%$ & $\begin{array}{l}\text { Convenie } \\
\mathrm{nt} \\
\text { sampling }\end{array}$ & $\begin{array}{l}69.6 \% \text { reported at least one sleep } \\
\text { difficulty and frequent awakenings was } \\
\text { the most prevalent problem. They were } \\
\text { associated with home confinement } \\
\text { without working, female gender and } \\
\text { diagnosed or suspected SDB. Older age } \\
\text { was protective factor. }\end{array}$ \\
\hline $\begin{array}{l}\text { Qi et } \\
\text { al., } \\
2020\end{array}$ & $\begin{array}{l}\text { Cross- } \\
\text { section } \\
\text { al }\end{array}$ & $\begin{array}{l}\text { Hospita } \\
1\end{array}$ & $\begin{array}{l}\text { Gradu } \\
\text { ate }\end{array}$ & China & $\begin{array}{l}\text { Medica } \\
1 \\
\text { worker } \\
\mathrm{s}\end{array}$ & $\begin{array}{l}33.1 \pm \\
8.4\end{array}$ & $\begin{array}{l}\text { Insomnia, } \\
\text { Anxiety }\end{array}$ & $\begin{array}{l}\text { Onlin } \\
\mathrm{e}\end{array}$ & $\begin{array}{l}\text { PSQI, } \\
\text { AIS }\end{array}$ & 6 & 3061 & $19.60 \%$ & $\begin{array}{l}\text { Convenie } \\
\mathrm{nt} \\
\text { sampling }\end{array}$ & $\begin{array}{l}\text { Compared to non-Frontline Medical } \\
\text { Workers, FMW had significantly } \\
\text { higher scores of PSQI }(9.3 \pm 3.8 \text { vs } 7.5 \\
\pm 3.7 ; \mathrm{P}<0.001 ;) \text {, AIS }(6.9 \pm 4.3 \text { vs } \\
5.3 \pm 3.8 ; \mathrm{P}<0.001 ;) \text {, higher sleep } \\
\text { disturbance according to }(78.4 \% \text { vs } \\
61.0 \% \text {; relative risk }[\mathrm{RR}] 1 / 41.29 ; \mathrm{P}< \\
0.001) \text { and AIS }>6 \text { points }(51.7 \% \text { vs } \\
35.6 \% \text {; RR } 1 / 41.45 ; \mathrm{P}<0.001) .\end{array}$ \\
\hline $\begin{array}{l}\text { Que et } \\
\text { al., } \\
2020\end{array}$ & $\begin{array}{l}\text { Cross- } \\
\text { section } \\
\text { al }\end{array}$ & $\begin{array}{l}\text { Hospita } \\
1\end{array}$ & $\begin{array}{l}\text { Gradu } \\
\text { ate }\end{array}$ & China & $\begin{array}{l}\text { Medica } \\
1 \\
\text { worker } \\
\mathrm{s}\end{array}$ & $\begin{array}{l}31.06 \\
\pm 6.99 \\
\text { years }\end{array}$ & $\begin{array}{l}\text { Insomnia, } \\
\text { Anxiety, } \\
\text { depressio } \\
\mathrm{n}\end{array}$ & $\begin{array}{l}\text { Onlin } \\
\mathrm{e}\end{array}$ & ISI & $>15$ & 2285 & $30.94 \%$ & $\begin{array}{l}\text { Convenie } \\
\mathrm{nt} \\
\text { sampling }\end{array}$ & $\begin{array}{l}\text { Prevalence of insomnia among HCW } \\
\text { was } 28.75 \% \text {. Being in the frontline } \\
\text { attention to negative or neutral } \\
\text { information about the pandemic, } \\
\text { receiving negative feedback from } \\
\text { families and friends who joined front- } \\
\text { line work, and unwillingness to join } \\
\text { front-line work if given a free choice } \\
\text { were three major factors for these } \\
\text { problems. }\end{array}$ \\
\hline $\begin{array}{l}\text { Romer } \\
\text { o- } \\
\text { Blanco } \\
\text { et al., } \\
2020 \\
\end{array}$ & $\begin{array}{l}\text { Longit } \\
\text { udinal } \\
\text { observa } \\
\text { tional } \\
\text { study } \\
\end{array}$ & $\begin{array}{l}\text { Hospita } \\
1\end{array}$ & $\begin{array}{l}\text { Bache } \\
\text { lor }\end{array}$ & Spain & $\begin{array}{l}\text { Nursin } \\
\mathrm{g} \\
\text { student } \\
\mathrm{s}\end{array}$ & $\begin{array}{l}20.6 \pm \\
4.62\end{array}$ & Insomnia & $\begin{array}{l}\text { Onlin } \\
\mathrm{e}\end{array}$ & PSQI & $>7$ & 207 & 19.4 & $\begin{array}{l}\text { Convenie } \\
\text { nt } \\
\text { sampling }\end{array}$ & $\begin{array}{l}\text { The mean PSQI score worsened by } \\
0.91 \text {. Sleep latency, sleep duration and } \\
\text { sleep efficiency were most affected by } \\
\text { lockdown. }\end{array}$ \\
\hline $\begin{array}{l}\text { Tan et } \\
\text { al., } \\
2020\end{array}$ & $\begin{array}{l}\text { Cross- } \\
\text { section } \\
\text { al }\end{array}$ & $\begin{array}{l}\text { Comm } \\
\text { unity }\end{array}$ & $\begin{array}{l}\text { Bache } \\
\text { lor }\end{array}$ & China & $\begin{array}{l}\text { Returni } \\
\text { ng } \\
\text { worker } \\
\mathrm{s}\end{array}$ & $\begin{array}{l}30.8 \pm \\
7.4 \mathrm{~s}\end{array}$ & $\begin{array}{l}\text { Insomnia, } \\
\text { Anxiety, } \\
\text { PTSD, } \\
\text { Depressio } \\
\text { n, Stress }\end{array}$ & $\begin{array}{l}\text { Onlin } \\
\mathrm{e}\end{array}$ & ISI & $>7$ & 673 & $74.40 \%$ & $\begin{array}{l}\text { Convenie } \\
\text { nt } \\
\text { sampling }\end{array}$ & $\begin{array}{l}2.3 \% \text { respondent reported of insomnia. } \\
\text { Factors that were associated with the } \\
\text { severity of psychiatric } \\
\text { symptoms in the workforce were } \\
\text { marital status (being single), presence } \\
\text { of physical symptom, poor physical } \\
\text { health and viewing return to work as a } \\
\text { health hazard (p<0.005). experience of } \\
\text { returning to work during the COVID- } \\
19 \text { pandemic did not confer an increase } \\
\text { in the prevalence of PTSD symptoms, } \\
\text { depression, anxiety and stress }\end{array}$ \\
\hline $\begin{array}{l}\text { Tselebi } \\
\text { s et al., } \\
2020\end{array}$ & $\begin{array}{l}\text { Cross- } \\
\text { section } \\
\text { al }\end{array}$ & $\begin{array}{l}\text { Hospita } \\
1\end{array}$ & $\begin{array}{l}\text { Gradu } \\
\text { ate }\end{array}$ & Greece & $\begin{array}{l}\text { Nursin } \\
\text { g staff }\end{array}$ & $\begin{array}{l}42.29 \\
\pm 10.7 \\
3\end{array}$ & $\begin{array}{l}\text { Insomnia, } \\
\text { Stress }\end{array}$ & $\begin{array}{l}\text { Onlin } \\
\mathrm{e}\end{array}$ & AIS & 6 & 150 & $20 \%$ & $\begin{array}{l}\text { Random } \\
\text { sampling }\end{array}$ & $\begin{array}{l}49.7 \% \text { patients reported of sleeping } \\
\text { difficulties. AIS scores were positively } \\
\text { correlated with stress and negatively } \\
\text { correlated with family support. } \\
\text { Perceived Stress Scale score and years } \\
\text { of experience were significant } \\
\text { predictors of AIS scores. }\end{array}$ \\
\hline
\end{tabular}




\begin{tabular}{|c|c|c|c|c|c|c|c|c|c|c|c|c|c|c|}
\hline $\begin{array}{l}\text { Turkog } \\
\text { lu et } \\
\text { al., } \\
2020\end{array}$ & $\begin{array}{l}\text { Pre- } \\
\text { post } \\
\text { study }\end{array}$ & $\begin{array}{l}\text { Hospita } \\
1\end{array}$ & $\begin{array}{l}\text { Schoo } \\
1\end{array}$ & Turkey & $\begin{array}{l}\text { Childre } \\
\mathrm{n} \text { with } \\
\text { ASD }\end{array}$ & $\begin{array}{l}7.89 \\
\text { years }\end{array}$ & $\begin{array}{l}\text { Sleep } \\
\text { disorder }\end{array}$ & $\begin{array}{l}\text { Face } \\
\text { to } \\
\text { face }\end{array}$ & CSHQ & $<23$ & 46 & $82.00 \%$ & $\begin{array}{l}\text { Convenie } \\
\text { nt } \\
\text { sampling }\end{array}$ & $\begin{array}{l}\text { Sleep problems increased among } \\
\text { children with ASD during home } \\
\text { confinement. Severity of the problems } \\
\text { were positively correlated with the } \\
\text { severity of ASD symptoms. }\end{array}$ \\
\hline $\begin{array}{l}\text { Amerio } \\
\text { et al., } \\
2020\end{array}$ & $\begin{array}{l}\text { Web } \\
\text { based } \\
\text { Cross- } \\
\text { section } \\
\text { al }\end{array}$ & $\begin{array}{l}\text { Healthc } \\
\text { are/Hos } \\
\text { pital }\end{array}$ & $\begin{array}{l}\text { Gradu } \\
\text { ate } \\
\text { and } \\
\text { above }\end{array}$ & Italy & $\begin{array}{l}\text { Healthc } \\
\text { are } \\
\text { Provide } \\
\text { rs }\end{array}$ & $\begin{array}{l}52.31 \\
\pm \\
12.24\end{array}$ & $\begin{array}{l}\text { Insomnia, } \\
\text { generalize } \\
\text { d anxiety } \\
\text { disorder }\end{array}$ & $\begin{array}{l}\text { Onlin } \\
\text { e } \\
\text { Surve } \\
\text { y }\end{array}$ & ISI & $>7$ & 131 & $51.90 \%$ & $\begin{array}{l}\text { Convenie } \\
\text { nt } \\
\text { sampling }\end{array}$ & $\begin{array}{l}\text { Sleep problems were detected in } 37.6 \% \\
\text { of the participants. ISI was higher in } \\
\text { female. Urban citizens scorer higher } \\
\text { than rural residents. Other risk factors } \\
\text { were intolerance to uncertainty, living } \\
\text { in the pandemic, unaware of multiple } \\
\text { aspects of this novel situation, } \\
\text { loneliness, COVID-19 related worry, } \\
\text { lack of opportunities of physical } \\
\text { activity and exposure to natural } \\
\text { environment. }\end{array}$ \\
\hline $\begin{array}{l}\text { Bargha } \\
\text { mi et } \\
\text { al., } \\
2020\end{array}$ & $\begin{array}{l}\text { Cross- } \\
\text { section } \\
\text { al }\end{array}$ & $\begin{array}{l}\text { Hospita } \\
1 \\
\text { (outdoo } \\
\mathrm{r} \text { and } \\
\text { indoor) }\end{array}$ & $\begin{array}{l}\text { Not } \\
\text { report } \\
\text { ed }\end{array}$ & Iran & $\begin{array}{l}\text { COVI } \\
\text { D-19 } \\
\text { Patient } \\
\text { s }\end{array}$ & $\begin{array}{l}40.34 \\
\pm 14.3 \\
9 \\
\text { (hospi } \\
\text { talize } \\
d \\
\text { patien } \\
\text { ts) } \\
\text { and } \\
43.62 \\
\pm \\
15.81( \\
\text { non- } \\
\text { hospit } \\
\text { alized } \\
\text { patien } \\
\text { ts) }\end{array}$ & $\begin{array}{l}\text { Insomnia, } \\
\text { general } \\
\text { anxiety } \\
\text { disorder, } \\
\text { major } \\
\text { depressive } \\
\text { disorder, } \\
\text { adjustmen } \\
\text { t disorder }\end{array}$ & $\begin{array}{l}\text { Mixe } \\
\mathrm{d} \\
\text { (onlin } \\
\mathrm{e} \text { and } \\
\text { phone } \\
\text { interv } \\
\text { iew) }\end{array}$ & $\begin{array}{l}\text { Perceived } \\
\text { Stress } \\
\text { Scale-14 } \\
\text { (PSS 14) } \\
\text { questionn } \\
\text { aires }\end{array}$ & $>5$ & 82 & $39.03 \%$ & $\begin{array}{l}\text { Convenie } \\
\text { nt } \\
\text { sampling }\end{array}$ & $\begin{array}{l}\text { Prevalence of insomnia } 61.6 \% \text {. The } \\
\text { prevalence of sleep disorders was } \\
\text { higher among the frontline healthcare } \\
\text { workers compared to the non-frontline } \\
\text { and non- medical staff. Risk factors } \\
\text { were medical occupation, family } \\
\text { burden, bereavement, anxiety, and } \\
\text { depression }\end{array}$ \\
\hline $\begin{array}{l}\text { Cai et } \\
\text { al., } \\
2020\end{array}$ & $\begin{array}{l}\text { Case- } \\
\text { control }\end{array}$ & $\begin{array}{l}\text { Hospita } \\
1 / \\
\text { Healthc } \\
\text { are }\end{array}$ & $\begin{array}{l}\text { Gradu } \\
\text { ate } \\
\text { and } \\
\text { above }\end{array}$ & China & $\begin{array}{l}\text { Frontli } \\
\text { ne and } \\
\text { non } \\
\text { frontlin } \\
\text { e } \\
\text { medica } \\
1 \\
\text { worker } \\
\text { s }\end{array}$ & $\begin{array}{l}30.5 \pm \\
8.7 \\
\text { (non } \\
\text { frontli } \\
\text { ne) } \\
\text { and } \\
30.6 \pm \\
8.8 \\
\text { (frontl } \\
\text { ine) }\end{array}$ & $\begin{array}{l}\text { Insomnia, } \\
\text { anxiety } \\
\text { and } \\
\text { depressive } \\
\text { symptoms }\end{array}$ & $\begin{array}{l}\text { Onlin } \\
\text { e } \\
\text { Surve } \\
y\end{array}$ & ISI & $>9$ & 2346 & $29.7 \%$ & $\begin{array}{l}\text { Convenie } \\
\text { nt } \\
\text { sampling }\end{array}$ & $\begin{array}{l}\text { Prevalence of insomnia was } 38 \% \\
\text { insomnia. Being an only child, } \\
\text { exposure to COVID-19 patients and } \\
\text { depression increase the risk of insomnia }\end{array}$ \\
\hline $\begin{array}{l}\text { Chandr } \\
\text { a et al., } \\
2020\end{array}$ & $\begin{array}{l}\text { Cross- } \\
\text { section } \\
\text { al }\end{array}$ & $\begin{array}{l}\text { Comm } \\
\text { unity }\end{array}$ & $\begin{array}{l}\text { Not } \\
\text { report } \\
\text { ed }\end{array}$ & Nepal & $\begin{array}{l}\text { Quaran } \\
\text { tined } \\
\text { people }\end{array}$ & $\begin{array}{l}29.5 \pm \\
9.77\end{array}$ & Insomnia & $\begin{array}{l}\text { Onlin } \\
\mathrm{e} \\
\text { surve } \\
\mathrm{y}\end{array}$ & & $>7$ & 206 & $53.40 \%$ & $\begin{array}{l}\text { Not } \\
\text { specified }\end{array}$ & $\begin{array}{l}\text { Prevalence of insomnia } 19.7 \% \text {. Being } \\
\text { female, married and current workload } \\
\text { and stress was associated with insomnia }\end{array}$ \\
\hline $\begin{array}{l}\text { Direnz } \\
\text { o et al., } \\
2020\end{array}$ & $\begin{array}{l}\text { web- } \\
\text { based }\end{array}$ & $\begin{array}{l}\text { Comm } \\
\text { unity }\end{array}$ & $\begin{array}{l}\text { Mixe } \\
d\end{array}$ & Italy & $\begin{array}{l}\text { Quaran } \\
\text { tined } \\
\text { people }\end{array}$ & $\begin{array}{l}48.5 \\
\text { years }\end{array}$ & $\begin{array}{l}\text { Insomnia, } \\
\text { depressed } \\
\text { mood, }\end{array}$ & $\begin{array}{l}\text { Electr } \\
\text { onic/ } \\
\text { Onlin }\end{array}$ & $\begin{array}{l}\text { Self - } \\
\text { designed }\end{array}$ & & 602 & $19.90 \%$ & $\begin{array}{c}\text { Not } \\
\text { specified }\end{array}$ & $\begin{array}{l}\text { The prevalence of insomnia disorder in } \\
\text { the whole sample was } 42.8 \% \text {. Risk } \\
\text { factors of insomnia was female }\end{array}$ \\
\hline
\end{tabular}




\begin{tabular}{|c|c|c|c|c|c|c|c|c|c|c|c|c|c|c|}
\hline & & & & & & & $\begin{array}{l}\text { hypochon } \\
\text { dria, } \\
\text { anxiety }\end{array}$ & $\begin{array}{l}\text { e } \\
\text { Surve } \\
\text { y }\end{array}$ & & & & & & $\begin{array}{l}\text { gender, younger age, and higher fatigue } \\
\text { and severe anxiety. }\end{array}$ \\
\hline $\begin{array}{l}\text { Jahram } \\
\text { i et al., } \\
2020\end{array}$ & $\begin{array}{l}\text { Cross } \\
\text { section } \\
\text { al }\end{array}$ & $\begin{array}{l}\text { Healthc } \\
\text { are }\end{array}$ & $\begin{array}{l}\text { Gradu } \\
\text { ate } \\
\text { and } \\
\text { above }\end{array}$ & Bahrain & $\begin{array}{l}\text { Healthc } \\
\text { are } \\
\text { worker } \\
\text { s }\end{array}$ & $\begin{array}{l}40.2 \pm \\
9.7\end{array}$ & $\begin{array}{l}\text { Insomnia, } \\
\text { stress }\end{array}$ & $\begin{array}{l}\text { Onlin } \\
\mathrm{e} \\
\text { Surve } \\
\mathrm{y}\end{array}$ & PSQI & $\geq 5$ & 257 & $30 \%$ & $\begin{array}{l}\text { Purposive } \\
\text { sampling }\end{array}$ & $\begin{array}{l}\text { Duration of sleep increased on } \\
\text { weekdays (Baseline }=7.9 \pm 1.0 \mathrm{~h} \text {, Stay- } \\
\text { at-Home }=8.4 \pm 1.1 \mathrm{~h}, \mathrm{p}<0.0001 \text { ) and } \\
\text { weekends }(8.4 \pm 1.5 \mathrm{~h}, 8.8 \pm 1.2 \mathrm{~h}, \mathrm{p}< \\
0.05 \text { ) during Stay-at-Home }\end{array}$ \\
\hline $\begin{array}{l}\text { K Zhuo } \\
\text { et al., } \\
2020\end{array}$ & $\begin{array}{l}\text { Cohort } \\
\text { study }\end{array}$ & $\begin{array}{l}\text { Hospita } \\
1\end{array}$ & $\begin{array}{l}\text { Gradu } \\
\text { ate } \\
\text { and } \\
\text { above }\end{array}$ & China & $\begin{array}{l}\text { Medica } \\
1 \text { and } \\
\text { nursing } \\
\text { staff }\end{array}$ & 41.92 & Insomnia & $\begin{array}{l}\text { Mixe } \\
\mathrm{d} \\
\text { (onlin } \\
\text { e and } \\
\text { phone } \\
\text { interv } \\
\text { iew) }\end{array}$ & ISI & $>7$ & 26 & $53.84 \%$ & $\begin{array}{l}\text { Convenie } \\
\text { nt } \\
\text { sampling }\end{array}$ & $\begin{array}{l}\text { Levels of social support for medical } \\
\text { staff were significantly associated with } \\
\text { sleep quality }\end{array}$ \\
\hline $\begin{array}{l}\text { Killgor } \\
\text { e et al., } \\
2020\end{array}$ & $\begin{array}{l}\text { Cross- } \\
\text { section } \\
\text { al }\end{array}$ & $\begin{array}{l}\text { Comm } \\
\text { unity }\end{array}$ & $\begin{array}{l}\text { Not } \\
\text { report } \\
\text { ed }\end{array}$ & $\begin{array}{l}\text { United } \\
\text { States }\end{array}$ & $\begin{array}{l}\text { Quaran } \\
\text { tined } \\
\text { people }\end{array}$ & 26.5 & Insomnia & $\begin{array}{l}\text { Onlin } \\
\mathrm{e}\end{array}$ & ISI & $>7$ & 1013 & $44.03 \%$ & $\begin{array}{l}\text { Not } \\
\text { specified }\end{array}$ & $\begin{array}{l}\text { Higher level of social capital was } \\
\text { positively associated with increased } \\
\text { quality of sleep but anxiety and stress } \\
\text { reduced the positive effects of social } \\
\text { capital on sleep quality }\end{array}$ \\
\hline $\begin{array}{l}\text { Korkm } \\
\text { az et } \\
\text { al., } \\
2020\end{array}$ & $\begin{array}{l}\text { Cohort } \\
\text { study }\end{array}$ & $\begin{array}{l}\text { Hospita } \\
1\end{array}$ & $\begin{array}{l}\text { Gradu } \\
\text { ate } \\
\text { and } \\
\text { above }\end{array}$ & Turkey & $\begin{array}{l}\text { Healthc } \\
\text { are } \\
\text { Worker } \\
\mathrm{s}\end{array}$ & $\begin{array}{l}30.7 \pm \\
6.2 \\
(\text { fema } \\
\text { le) } \\
35.6 \pm \\
8.7 \\
\text { (male }\end{array}$ & $\begin{array}{l}\text { Insomnia, } \\
\text { anxiety } \\
\text { and } \\
\text { depressio } \\
\mathrm{n} \\
\text { symptoms }\end{array}$ & $\begin{array}{l}\text { Face } \\
\text { to } \\
\text { Face/ } \\
\text { Onlin } \\
\text { e/ } \\
\text { Recor } \\
\text { ds } \\
\text { data }\end{array}$ & PSQI & $\geq 5$ & 140 & $56 \%$ & $\begin{array}{l}\text { Not } \\
\text { specified }\end{array}$ & $\begin{array}{l}\text { A total of } 76.7 \% \text { of the medically } \\
\text { isolated population reported difficulty } \\
\text { falling asleep while the corresponding } \\
\text { rates among self-reported isolation } \\
(51.0 \%) \text { and non-reported isolation } \\
\text { groups ( } 42.3 \% \text { ) were significantly } \\
\text { lower. Risk factors: Fear of infection, } \\
\text { isolation }\end{array}$ \\
\hline $\begin{array}{l}\text { Nallebe } \\
\text { lle et } \\
\text { al., } \\
2020\end{array}$ & $\begin{array}{l}\text { Cohort } \\
\text { study }\end{array}$ & $\begin{array}{l}\text { Individ } \\
\text { ual }\end{array}$ & $\begin{array}{l}\text { Not } \\
\text { report } \\
\text { ed }\end{array}$ & $\begin{array}{l}\text { United } \\
\text { States }\end{array}$ & $\begin{array}{l}\text { COVI } \\
\text { D-19 } \\
\text { patients }\end{array}$ & 49 & $\begin{array}{l}\text { Neuropsy } \\
\text { chiatric } \\
\text { manifestat } \\
\text { ions }\end{array}$ & N/A & None & N/A & $\begin{array}{r}40,46 \\
9\end{array}$ & $45 \%$ & $\begin{array}{l}\text { Not } \\
\text { specified }\end{array}$ & $\begin{array}{l}\text { The prevalence of insomnia among } \\
\text { participants was } 52.8 \% \text { which was } \\
\text { predicted by gender, working } \\
\text { experience, chronic diseases, midday } \\
\text { nap duration, direct participation in } \\
\text { rescue of patients with COVID-19, } \\
\text { frequency of night shifts, professional } \\
\text { psychological assistance during the } \\
\text { pandemic, negative experiences (such } \\
\text { as family, friends, or colleagues being } \\
\text { seriously ill or dying due to COVID- } \\
\text { 19), the degree of fear of COVID-19, } \\
\text { fatigue, and stress }\end{array}$ \\
\hline $\begin{array}{l}\text { Patra et } \\
\text { al., } \\
2020\end{array}$ & $\begin{array}{l}\text { Observ } \\
\text { ational, } \\
\text { Cross } \\
\text { section } \\
\text { al }\end{array}$ & $\begin{array}{l}\text { Individ } \\
\text { ual }\end{array}$ & $\begin{array}{l}\text { Mixe } \\
d\end{array}$ & India & $\begin{array}{l}\text { Quaran } \\
\text { tined } \\
\text { people }\end{array}$ & $\begin{array}{l}\text { Group } \\
\text { A: } \\
53.44, \\
\text { Group } \\
\text { B:52. } \\
76\end{array}$ & $\begin{array}{l}\text { Stress, } \\
\text { insomnia }\end{array}$ & $\begin{array}{l}\text { Face } \\
\text { to } \\
\text { Face/ } \\
\text { Onlin } \\
\text { e/ } \\
\text { Recor } \\
\text { ds } \\
\text { data }\end{array}$ & PSQI & $>5$ & 100 & $\begin{array}{l}\text { Group } \\
\text { A: } \\
30 \%, \\
\text { Group } \\
\text { B: } 50 \%\end{array}$ & $\begin{array}{l}\text { Random } \\
\text { sampling }\end{array}$ & $\begin{array}{l}\text { Prevalence of poor sleep quality was } \\
14.9 \% \text {. The average PSQI score was } \\
3.0 \pm 2.5 \text { and average duration of sleep } \\
\text { was } 7.6 \pm 1.2 \mathrm{~h} . . \text { Older than } 24 \text { years, } \\
\text { higher education level, negative attitude } \\
\text { towards COVID- } 19 \text { control measures, } \\
\text { anxiety and depression was related to } \\
\text { poor sleep. . }\end{array}$ \\
\hline
\end{tabular}




\begin{tabular}{|c|c|c|c|c|c|c|c|c|c|c|c|c|c|c|}
\hline $\begin{array}{l}\text { Salehin } \\
\text { ejad et } \\
\text { al., } \\
2020\end{array}$ & $\begin{array}{l}\text { Cross } \\
\text { section } \\
\text { al }\end{array}$ & $\begin{array}{l}\text { individ } \\
\text { ual }\end{array}$ & $\begin{array}{l}\text { Not } \\
\text { report } \\
\text { ed }\end{array}$ & Iran & $\begin{array}{l}\text { Quaran } \\
\text { tined } \\
\text { people }\end{array}$ & $\begin{array}{l}25.79 \\
\pm 7.31\end{array}$ & Insomnia & online & PSQI & 3 & 160 & $14.38 \%$ & & $\begin{array}{l}\text { During the period of isolation patients } \\
\text { of chronic insomnia, the average score } \\
\text { of PSQI was } 11.28 \text { which was much } \\
\text { higher than the baseline score, } \\
\text { indicating worse sleep quality, higher } \\
\text { anxiety scores and increased severity of } \\
\text { somatic symptoms during the } \\
\text { pandemic. }\end{array}$ \\
\hline $\begin{array}{l}\text { Song et } \\
\text { al., } \\
2020\end{array}$ & $\begin{array}{l}\text { cross- } \\
\text { section } \\
\text { al }\end{array}$ & $\begin{array}{l}\text { Comm } \\
\text { unity }\end{array}$ & $\begin{array}{l}\text { Mixe } \\
d\end{array}$ & China & $\begin{array}{l}\text { Genera } \\
1 \\
\text { Populat } \\
\text { ion }\end{array}$ & 35.35 & $\begin{array}{l}\text { Insomnia, } \\
\text { depressio } \\
\text { n, somatic } \\
\text { symptoms }\end{array}$ & online & ISI & $\geq 8$ & 709 & $25.80 \%$ & $\begin{array}{l}\text { Convenie } \\
\mathrm{nt} \\
\text { sampling }\end{array}$ & $\begin{array}{l}\text { The prevalence of current insomnia (ISI } \\
\text { score of } \geq 10 \text { ) was } 29.9 \% \text {. The weighted } \\
\text { prevalence of worsened sleep quality, } \\
\text { difficulty in sleep initiation, and } \\
\text { shortened sleep duration since the } \\
\text { outbreak were } 38.3 \%, 29.8 \% \text {, and } \\
29.1 \% \text {, respectively. Insufficient stock } \\
\text { of masks was significantly associated } \\
\text { with worsened sleep quality, impaired } \\
\text { sleep initiation, shortened sleep } \\
\text { duration, and current insomnia. }\end{array}$ \\
\hline $\begin{array}{l}\text { Stojano } \\
\text { v et al., } \\
2020\end{array}$ & $\begin{array}{l}\text { Cross- } \\
\text { section } \\
\text { al }\end{array}$ & $\begin{array}{l}\text { Clinica } \\
1 \text { center }\end{array}$ & $\begin{array}{l}\text { Mixe } \\
\text { d }\end{array}$ & Siberia & $\begin{array}{l}\text { Healthc } \\
\text { are } \\
\text { worker }\end{array}$ & $\begin{array}{l}\text { Group } \\
1- \\
39.1 \pm \\
7.3 \\
\text { Group } \\
2- \\
42.5 \pm \\
9.7\end{array}$ & $\begin{array}{l}\text { Insomnia, } \\
\text { generalize } \\
\text { d anxiety } \\
\text { disorder, } \\
\text { Depressio } \\
\text { n }\end{array}$ & $\begin{array}{l}\text { Onlin } \\
\mathrm{e}\end{array}$ & PSQI & $>7$ & 201 & $\begin{array}{l}\text { not } \\
\text { reporte } \\
d\end{array}$ & $\begin{array}{l}\text { Not } \\
\text { specified }\end{array}$ & $\begin{array}{l}\text { Prevalence of sleep disturbance was } \\
55.6 \% \text {. Compared to the previous } \\
\text { sample, the preschoolers during the } \\
\text { pandemic reported later bedtime and } \\
\text { waking time, longer nocturnal sleep } \\
\text { and shorter nap. Proper sleeping } \\
\text { arrangement reduced electronic device } \\
\text { use, harmonious family environment } \\
\text { and increased interaction with parents } \\
\text { were associated with less sleep } \\
\text { disturbance. }\end{array}$ \\
\hline $\begin{array}{l}\text { Z Li et } \\
\text { al., } \\
2020\end{array}$ & $\begin{array}{l}\text { Cohort } \\
\text { study }\end{array}$ & $\begin{array}{l}\text { Hospita } \\
1\end{array}$ & $\begin{array}{l}\text { Not } \\
\text { report } \\
\text { ed }\end{array}$ & China & $\begin{array}{l}\text { COVI } \\
\text { D-19 } \\
\text { patients }\end{array}$ & 50.5 & $\begin{array}{l}\text { Sleep } \\
\text { disorder, } \\
\text { respirator } \\
\text { y } \\
\text { disfunctio } \\
\text { n, anxiety, } \\
\text { depressio } \\
\text { n }\end{array}$ & $\begin{array}{l}\text { Face } \\
\text { to } \\
\text { face }\end{array}$ & $\begin{array}{l}\text { designed } \\
\text { questionn } \\
\text { aire }\end{array}$ & & 280 & $51.80 \%$ & $\begin{array}{l}\text { Convenie } \\
\mathrm{nt} \\
\text { sampling }\end{array}$ & $\begin{array}{l}\text { Compared with nonmedical health } \\
\text { workers, health care workers had a } \\
\text { higher prevalence of insomnia ( } 38.4 \text { vs. } \\
30.5 \%) \text {. Among medical health } \\
\text { workers, having organic disease was an } \\
\text { independent factor for insomnia. Living } \\
\text { in rural areas, being female, and being } \\
\text { at risk of contact with COVID-19 } \\
\text { patients were the most common risk } \\
\text { factors for insomnia ( }<<0.01 \text { or } 0.05 \text { ). }\end{array}$ \\
\hline $\begin{array}{l}\text { Zhao et } \\
\text { al., } \\
2020\end{array}$ & $\begin{array}{l}\text { Cross- } \\
\text { section } \\
\text { al }\end{array}$ & $\begin{array}{l}\text { Healthc } \\
\text { are }\end{array}$ & $\begin{array}{l}\text { Not } \\
\text { report } \\
\text { ed }\end{array}$ & China & $\begin{array}{l}\text { Healthc } \\
\text { are } \\
\text { provide } \\
\text { rs }\end{array}$ & $\begin{array}{r}\text { not } \\
\text { report } \\
\text { ed }\end{array}$ & $\begin{array}{l}\text { Insomnia, } \\
\text { anxiety, } \\
\text { depressio } \\
\text { n }\end{array}$ & online & ISI & & 972 & $\begin{array}{r}\text { Not } \\
\text { reporte } \\
\mathrm{d}\end{array}$ & $\begin{array}{l}\text { Not } \\
\text { specified }\end{array}$ & $\begin{array}{l}\text { Around one-third }(36.38 \%) \text { of } \\
\text { participants reported of poor sleep } \\
\text { during the COVID-19 pandemic. } \\
\text { Higher perceived stress was } \\
\text { significantly associated with higher } \\
\text { anxiety levels, which, in turn, } \\
\text { was associated with lower sleep } \\
\text { quality. Self-esteem moderated the } \\
\text { indirect effect of perceived stress on } \\
\text { sleep quality }\end{array}$ \\
\hline
\end{tabular}




\begin{tabular}{|c|c|c|c|c|c|c|c|c|c|c|c|c|c|c|}
\hline $\begin{array}{l}\text { Zhi- } \\
\text { hao Tu } \\
\text { et al., } \\
2020\end{array}$ & $\begin{array}{l}\text { Cross- } \\
\text { section } \\
\text { al }\end{array}$ & $\begin{array}{l}\text { Healthc } \\
\text { are }\end{array}$ & $\begin{array}{l}\text { Mixe } \\
d\end{array}$ & China & $\begin{array}{l}\text { Healthc } \\
\text { are } \\
\text { provide } \\
\text { rs }\end{array}$ & 34.44 & $\begin{array}{l}\text { Insomnia, } \\
\text { anxiety }\end{array}$ & $\begin{array}{l}\text { Onlin } \\
\mathrm{e}\end{array}$ & PSQI & $\geq 7$ & 100 & & $\begin{array}{l}\text { Cluster } \\
\text { sampling }\end{array}$ & $\begin{array}{l}\text { Prevalence of insomnia was } 23.3 \% \\
\text { during the COVID- } 19 \text { pandemic. } \\
\text { Female gender, urban dweller and } \\
\text { having depression was risk factors of } \\
\text { insomnia. }\end{array}$ \\
\hline $\begin{array}{l}\text { Voitsisi } \\
\text { s et al., } \\
2020\end{array}$ & $\begin{array}{l}\text { Cross- } \\
\text { section } \\
\text { al }\end{array}$ & $\begin{array}{l}\text { Comm } \\
\text { unity }\end{array}$ & $\begin{array}{l}\text { Not } \\
\text { report } \\
\text { ed }\end{array}$ & Greece & $\begin{array}{l}\text { Greek } \\
\text { Populat } \\
\text { ion }\end{array}$ & $\begin{array}{l}\text { Not } \\
\text { report } \\
\text { ed }\end{array}$ & Insomnia & $\begin{array}{l}\text { Onlin } \\
\mathrm{e}\end{array}$ & AIS. & $\begin{array}{l}\text { Not } \\
\text { repo } \\
\text { rted }\end{array}$ & 2,427 & $23.80 \%$ & $\begin{array}{c}\text { Not } \\
\text { specified }\end{array}$ & $\begin{array}{l}\text { Compared to pre-pandemic time, the } \\
\text { severity of insomnia was less during } \\
\text { the lockdown period (ISI score } 6.6 \mathrm{vs} \\
8.1 \text { ) and significantly less participants } \\
\text { reported of insomnia ( } 34.4 \% \text { ) vs } \\
50.3 \% \text { ). }\end{array}$ \\
\hline $\begin{array}{l}\text { W } \\
\text { Wang } \\
\text { et al., } \\
2020\end{array}$ & $\begin{array}{l}\text { cross- } \\
\text { section } \\
\text { al }\end{array}$ & $\begin{array}{l}\text { Healthc } \\
\text { are }\end{array}$ & $\begin{array}{l}\text { Mixe } \\
d\end{array}$ & China & $\begin{array}{l}\text { Healthc } \\
\text { are } \\
\text { worker } \\
\mathrm{s} \text { (non- } \\
\text { medica } \\
1 \text { staff, } \\
\text { frontlin } \\
\text { e and } \\
\text { non- } \\
\text { frontlin } \\
\text { e } \\
\text { worker } \\
\text { s) }\end{array}$ & 41.5 & $\begin{array}{l}\text { Insomnia, } \\
\text { anxiety, } \\
\text { depressio } \\
\text { n }\end{array}$ & $\begin{array}{l}\text { Onlin } \\
\mathrm{e}\end{array}$ & PSQI & $\geq 5$ & 2737 & $35.50 \%$ & $\begin{array}{l}\text { Convenie } \\
\text { nt } \\
\text { sampling }\end{array}$ & $\begin{array}{l}\text { Prevalence of insomnia was } \\
\text { significantly higher among PHQ- } 9 \geq 10 \\
\text { GPs compared to PHQ-9 }<10 \text {. Insomnia } \\
11.60 \pm 5.53 \text { vs. } 4.84 \pm 3.81 ; \mathrm{p}<.001 \text { ) } \\
\text { Risk Factors: Spending more than } 3 \\
\text { hours searching for Covid-19 related } \\
\text { information, Inadequate PPEs, Visiting } \\
\text { more Covid-19 infected patients. } \\
\text { Interventions: providing continuity of } \\
\text { care for patients at the community- } \\
\text { level, adequate PPE to GPs and a clear } \\
\text { guidance from public health } \\
\text { institutions. }\end{array}$ \\
\hline $\begin{array}{l}\text { Wang } \\
\text { et al., } \\
2020\end{array}$ & $\begin{array}{l}\text { Cross- } \\
\text { section } \\
\text { al }\end{array}$ & $\begin{array}{l}\text { Childre } \\
\mathrm{n} \\
\text { Healthc } \\
\text { are } \\
\text { Centre }\end{array}$ & $\begin{array}{l}\text { Mixe } \\
d\end{array}$ & China & $\begin{array}{l}\text { Healthc } \\
\text { are } \\
\text { worker } \\
\text { s } \\
\text { (Doctor } \\
\text { Nurse) }\end{array}$ & $\begin{array}{l}33.75 \\
\pm 8.41\end{array}$ & $\begin{array}{l}\text { Insomnia, } \\
\text { anxiety, } \\
\text { depressio } \\
\text { n }\end{array}$ & $\begin{array}{l}\text { Self- } \\
\text { report } \\
\text { ed }\end{array}$ & PSQI & $>7$ & 123 & $10 \%$ & $\begin{array}{l}\text { Convenie } \\
\text { nt } \\
\text { sampling }\end{array}$ & $\begin{array}{l}\text { Of the patients, } 29.3 \% \text { reported of } \\
\text { insomnia. Hospitalization and being } \\
\text { female were associated with higher risk } \\
\text { of insomnia. }\end{array}$ \\
\hline $\begin{array}{l}\text { Wang } \\
\text { et al., } \\
2020\end{array}$ & $\begin{array}{l}\text { Cross- } \\
\text { section } \\
\text { al }\end{array}$ & $\begin{array}{l}\text { Healthc } \\
\text { are }\end{array}$ & $\begin{array}{l}\text { Mixe } \\
\text { d }\end{array}$ & China & $\begin{array}{l}\text { Medica } \\
\text { 1 Staff }\end{array}$ & 37 & $\begin{array}{l}\text { Insomnia, } \\
\text { anxiety, } \\
\text { depressio } \\
\mathrm{n}\end{array}$ & $\begin{array}{l}\text { Onlin } \\
\mathrm{e}\end{array}$ & PSQI. & $>5$ & 274 & $22.60 \%$ & $\begin{array}{c}\text { Not } \\
\text { specified }\end{array}$ & $\begin{array}{l}\text { Frontline medical workers had higher } \\
\text { rates insomnia ( } 47.8 \% \text { vs. } 29.1 \%) \text { than } \\
\text { non-frontline medical workers. }\end{array}$ \\
\hline $\begin{array}{l}\text { Wang } \\
\text { et al., } \\
2020\end{array}$ & $\begin{array}{l}\text { Cross } \\
\text { section } \\
\text { al }\end{array}$ & $\begin{array}{l}\text { Hospita } \\
1\end{array}$ & $\begin{array}{l}\text { Mixe } \\
d\end{array}$ & China & $\begin{array}{l}\text { COVI } \\
\text { D-19 } \\
\text { inpatie } \\
\text { nts }\end{array}$ & 52.5 & Insomnia & $\begin{array}{l}\text { Onlin } \\
\mathrm{e}\end{array}$ & CISI & $\geq 8$ & 484 & 49.8 & $\begin{array}{c}\text { Not } \\
\text { specified }\end{array}$ & $\begin{array}{l}\text { The sleep quality among Nepalese } \\
\text { residents varied significantly before and } \\
\text { after COVID-19 pandemic. Moderate } \\
\text { insomnia increased from } 2.9 \% \text { to } \\
16.5 \% \text {. After the pandemic } 11.7 \% \\
\text { reported of severe difficulty in falling } \\
\text { asleep, } 5.3 \% \text { had difficulty in sleep } \\
\text { maintenance, } 12.6 \% \text { had severe } \\
\text { awakening problem, } 16 \text { subjects were } \\
\text { dissatisfied with their sleep pattern, } \\
7.8 \% \text { of subjects had impaired quality } \\
\text { of life, } 3.9 \% \text { of subjects were worried } \\
\text { about their sleep problem. Female } \\
\text { gender and age <45 years was }\end{array}$ \\
\hline
\end{tabular}




\begin{tabular}{|c|c|c|c|c|c|c|c|c|c|c|c|c|c|c|}
\hline & & & & & & & & & & & & & & $\begin{array}{l}\text { associated with higher difficulties in } \\
\text { sleeping. }\end{array}$ \\
\hline $\begin{array}{l}\text { Wright } \\
\text { et al., } \\
2020\end{array}$ & $\begin{array}{l}\text { Observ } \\
\text { ational }\end{array}$ & $\begin{array}{l}\text { Univer } \\
\text { sity }\end{array}$ & $\begin{array}{l}\text { Not } \\
\text { report } \\
\text { ed }\end{array}$ & $\begin{array}{l}\text { United } \\
\text { States }\end{array}$ & $\begin{array}{l}\text { Univer } \\
\text { sity } \\
\text { student } \\
\text { s }\end{array}$ & $\begin{array}{r}22.2 \pm \\
1.7\end{array}$ & Insomnia & $\begin{array}{l}\text { Onlin } \\
\mathrm{e}\end{array}$ & & & 139 & $\begin{array}{r}\text { Not } \\
\text { reporte } \\
\mathrm{d}\end{array}$ & $\begin{array}{l}\text { Not } \\
\text { specified }\end{array}$ & Prevalence of insomnia was $52.2 \%$ \\
\hline $\begin{array}{l}\text { Xiao et } \\
\text { al., } \\
2020\end{array}$ & $\begin{array}{l}\text { Observ } \\
\text { ational, } \\
\text { cross- } \\
\text { section } \\
\text { al }\end{array}$ & $\begin{array}{l}\text { Hospita } \\
\text { 1/health } \\
\text { care }\end{array}$ & $\begin{array}{l}\text { Mixe } \\
d\end{array}$ & China & $\begin{array}{l}\text { Medica } \\
1 \text { Staff } \\
\text { (doctor } \\
\text { and } \\
\text { nurse) }\end{array}$ & $\begin{array}{l}32.31 \\
\pm 4.88\end{array}$ & $\begin{array}{l}\text { Insomnia, } \\
\text { anxiety }\end{array}$ & $\begin{array}{l}\text { Face } \\
\text { to } \\
\text { face }\end{array}$ & PSQI & $\begin{array}{l}\geq \\
20\end{array}$ & 180 & $28.30 \%$ & $\begin{array}{l}\text { Not } \\
\text { specified }\end{array}$ & $\begin{array}{l}\text { Prevalence of insomnia } 75 \% \text {. The } \\
\text { overall PSQI score was } 7.0 \pm 3.3 \text {. } \\
\text { Female sex and medical doctor were } \\
\text { risk factor of insomnia. }\end{array}$ \\
\hline $\begin{array}{l}\text { Xiao et } \\
\text { al., } \\
2020\end{array}$ & $\begin{array}{l}\text { Cross- } \\
\text { section } \\
\text { al }\end{array}$ & $\begin{array}{l}\text { Comm } \\
\text { unity }\end{array}$ & $\begin{array}{l}\text { Mixe } \\
\text { d }\end{array}$ & China & $\begin{array}{l}\text { Self- } \\
\text { isolated } \\
\text { individ } \\
\text { uals }\end{array}$ & $\begin{array}{l}37.78 \\
\pm 4.12\end{array}$ & $\begin{array}{l}\text { Insomnia, } \\
\text { anxiety }\end{array}$ & $\begin{array}{l}\text { Onlin } \\
\text { e }\end{array}$ & PSQI & $\begin{array}{l}\text { PSQ } \\
\text { I } \\
\text { total } \\
\text { scor } \\
\text { es } \geq \\
20\end{array}$ & 170 & $59.40 \%$ & $\begin{array}{l}\text { Not } \\
\text { specified }\end{array}$ & $\begin{array}{l}\text { Insomnia is related with severe sleep } \\
\text { apnea-hypopnea syndrome (SAHS). } \\
\text { ISI was positively correlated with total } \\
\text { sleep time (TST) and negatively } \\
\text { correlated with deep sleep; SRQ-20 } \\
\text { (OR= } 1.51 \text { ) and female sex (OR=1.15) } \\
\text { were risk factors for insomnia with } \\
\text { comorbid SAHS }\end{array}$ \\
\hline $\begin{array}{l}\text { Xingxi } \\
\text { ng et } \\
\text { al., } \\
2020\end{array}$ & $\begin{array}{l}\text { Cross- } \\
\text { section } \\
\text { al }\end{array}$ & $\begin{array}{l}\text { Healthc } \\
\text { are }\end{array}$ & $\begin{array}{l}\text { Mixe } \\
d\end{array}$ & China & $\begin{array}{l}\text { medica } \\
1 \text { staff } \\
\text { person } \\
\text { nel }\end{array}$ & $\begin{array}{l}\text { Not } \\
\text { report } \\
\text { ed }\end{array}$ & Insomnia & $\begin{array}{l}\text { Onlin } \\
\mathrm{e}^{-}\end{array}$ & AIS & $\begin{array}{l}\text { AIS } \\
\text { scor } \\
\text { e } \geq 6, \\
\text { SRQ } \\
-20 \\
\text { scor } \\
\mathrm{e} \geq 7\end{array}$ & $\begin{array}{l}948 \\
\text { volunt } \\
\text { eered } \\
\text { to } \\
\text { Wuha } \\
\mathrm{n}, 729 \\
\text { stayed } \\
\text { at } \\
\text { Ningb } \\
\text { o) }\end{array}$ & $22.02 \%$ & $\begin{array}{c}\text { Not } \\
\text { specified }\end{array}$ & $\begin{array}{l}56 \% \text { of participants reported of some } \\
\text { degree of insomnia, these levels are } \\
\text { notably higher than historically seen in } \\
\text { the general population. Greater worries } \\
\text { over COVID-19 were significantly } \\
\text { correlated with self-reported insomnia. } \\
\text { Insomnia severity was also } \\
\text { independently associated with elevated } \\
\text { suicidal ideation. }\end{array}$ \\
\hline $\begin{array}{l}\text { Xue et } \\
\text { al., } \\
2020 \\
\end{array}$ & $\begin{array}{l}\text { Cohort } \\
\text { study }\end{array}$ & $\begin{array}{l}\text { Comm } \\
\text { unity }\end{array}$ & $\begin{array}{l}\text { Mixe } \\
d\end{array}$ & China & $\begin{array}{l}\text { Quranti } \\
\text { ned } \\
\text { people }\end{array}$ & $\begin{array}{l}\text { Not } \\
\text { report } \\
\text { ed }\end{array}$ & Insomnia & $\begin{array}{l}\text { Onlin } \\
\mathrm{e}\end{array}$ & & & 707 & $\begin{array}{r}\text { Not } \\
\text { reporte } \\
\mathrm{d} \\
\end{array}$ & $\begin{array}{l}\text { Not } \\
\text { specified }\end{array}$ & Prevalence of insomnia $34 \%$ \\
\hline $\begin{array}{l}\text { Y Zhan } \\
\text { et al., } \\
2020\end{array}$ & $\begin{array}{l}\text { Cross- } \\
\text { section } \\
\text { al, } \\
\text { descrip } \\
\text { tive }\end{array}$ & $\begin{array}{l}\text { Healthc } \\
\text { are }\end{array}$ & $\begin{array}{l}\text { Not } \\
\text { report } \\
\text { ed }\end{array}$ & China & $\begin{array}{l}\text { frontlin } \\
\text { e } \\
\text { nurses } \\
\text { from } \\
\text { four } \\
\text { tertiary } \\
\text {-level } \\
\text { general } \\
\text { hospita } \\
\text { ls. } \\
\end{array}$ & $\begin{array}{l}\text { Not } \\
\text { report } \\
\text { ed }\end{array}$ & Insomnia & $\begin{array}{l}\text { Onlin } \\
\mathrm{e}\end{array}$ & AIS & $\begin{array}{l}\text { AIS } \\
\text { scor } \\
\mathrm{e} \geq 6\end{array}$ & 1794 & $3 \%$ & $\begin{array}{l}\text { Convenie } \\
\mathrm{nt} \\
\text { sampling }\end{array}$ & Prevalence of sleep disorder was $3.4 \%$ \\
\hline $\begin{array}{l}\text { Yang } \\
\text { et al., } \\
2020\end{array}$ & $\begin{array}{l}\text { Cross } \\
\text { section } \\
\text { al }\end{array}$ & $\begin{array}{l}\text { Comm } \\
\text { unity }\end{array}$ & $\begin{array}{l}\text { Mixe } \\
d\end{array}$ & China & $\begin{array}{l}\text { Workin } \\
\mathrm{g} \\
\text { individ } \\
\text { uals }\end{array}$ & $\begin{array}{r}36.3 \pm \\
9.1\end{array}$ & Insomnia & $\begin{array}{l}\text { Onlin } \\
\mathrm{e}^{-}\end{array}$ & CPSQI & $>5$ & 2,410 & $50.80 \%$ & $\begin{array}{l}\text { Convenie } \\
\text { nt } \\
\text { sampling }\end{array}$ & $\begin{array}{l}\text { Group chronic illness scored } \\
\text { significantly higher than control, } \\
\text { indicating poorer sleep quality among } \\
\text { them. }\end{array}$ \\
\hline $\begin{array}{l}\text { Yang } \\
\text { et al., } \\
2020\end{array}$ & $\begin{array}{l}\text { Cross- } \\
\text { section } \\
\text { al }\end{array}$ & $\begin{array}{l}\text { Individ } \\
\text { ual }\end{array}$ & $\begin{array}{l}\text { Mixe } \\
\text { d }\end{array}$ & China & $\begin{array}{l}\text { Insomn } \\
\text { ia } \\
\text { patients }\end{array}$ & $\begin{array}{l}\text { Not } \\
\text { report } \\
\text { ed }\end{array}$ & $\begin{array}{l}\text { Insomnia, } \\
\text { anxiety }\end{array}$ & $\begin{array}{l}\text { Onlin } \\
\mathrm{e}\end{array}$ & PSQI & & 764 & $21.50 \%$ & $\begin{array}{l}\text { Random } \\
\text { sampling }\end{array}$ & $\begin{array}{l}\text { Significant delay in bedtime, prolonged } \\
\text { average time to fall asleep and longer }\end{array}$ \\
\hline
\end{tabular}




\begin{tabular}{|c|c|c|c|c|c|c|c|c|c|c|c|c|c|c|}
\hline & & & & & & & & & & & & & & $\begin{array}{l}\text { average total duration of sleep during } \\
\text { quarantine was reported. }\end{array}$ \\
\hline $\begin{array}{l}\text { Yu et } \\
\text { al., } \\
2020\end{array}$ & $\begin{array}{l}\text { Cross } \\
\text { section } \\
\text { al }\end{array}$ & $\begin{array}{l}\text { Comm } \\
\text { nunity }\end{array}$ & $\begin{array}{l}\text { Not } \\
\text { report } \\
\text { ed }\end{array}$ & $\begin{array}{l}\text { Hong } \\
\text { kong }\end{array}$ & $\begin{array}{l}\text { Chines } \\
\text { e } \\
\text { populat } \\
\text { ion }\end{array}$ & $\begin{array}{l}\text { Not } \\
\text { report } \\
\text { ed }\end{array}$ & Insomnia & $\begin{array}{l}\text { Onlin } \\
\mathrm{e}\end{array}$ & ISI & $\begin{array}{l}5- \\
\text { poin } \\
\mathrm{t} \\
\text { Like } \\
\mathrm{rt} \\
\text { scale }\end{array}$ & 1138 & $\begin{array}{l}\text { Not } \\
\text { reporte } \\
\text { d }\end{array}$ & $\begin{array}{l}\text { Convenie } \\
\text { nt } \\
\text { sampling }\end{array}$ & $\begin{array}{l}\text { Prevalence of insomnia } 20.7 \% \text {. } \\
\text { Worrying about unemployment } \\
\text { negatively affected, and psychological } \\
\text { strengths (i.e., resilience and optimism) } \\
\text { positively affected sleep. }\end{array}$ \\
\hline $\begin{array}{l}\text { Z Liu } \\
\text { et al., } \\
2020\end{array}$ & $\begin{array}{l}\text { Pre- } \\
\text { post } \\
\text { study }\end{array}$ & & & & & & $\begin{array}{l}\text { Sleep } \\
\text { disorder }\end{array}$ & $\begin{array}{l}\text { Onlin } \\
\mathrm{e}\end{array}$ & CSHQ & & 1619 & & $\begin{array}{l}\text { Convenie } \\
\mathrm{nt} \\
\text { sampling }\end{array}$ & $\begin{array}{l}\text { Generalized anxiety and overall mental } \\
\text { health status were predictors of higher } \\
\text { score in PSQI indication higher severity } \\
\text { of insomnia. }\end{array}$ \\
\hline $\begin{array}{l}\text { Zhang } \\
\text { et al., } \\
2020\end{array}$ & $\begin{array}{l}\text { Cross- } \\
\text { section } \\
\text { al }\end{array}$ & $\begin{array}{l}\text { Healthc } \\
\text { are and } \\
\text { commu } \\
\text { nity }\end{array}$ & $\begin{array}{l}\text { Mixe } \\
\text { d }\end{array}$ & China & $\begin{array}{l}\text { Medica } \\
1 \\
\text { Health } \\
\text { worker } \\
\mathrm{s} \text { and } \\
\text { non } \\
\text { medica } \\
1 \\
\text { worker } \\
\mathrm{s}\end{array}$ & $\begin{array}{l}\text { Not } \\
\text { report } \\
\text { ed }\end{array}$ & $\begin{array}{l}\text { Insomnia, } \\
\text { anxiety, } \\
\text { somatic } \\
\text { disorder, } \\
\text { obsessive } \\
\text { compulsiv } \\
\text { e disorder }\end{array}$ & $\begin{array}{l}\text { Onlin } \\
\mathrm{e}\end{array}$ & ISI & $\begin{array}{l}\text { ISI } \\
\text { total } \\
\text { scor } \\
\mathrm{e}>8\end{array}$ & 2,182 & $35.80 \%$ & & Prevalence of sleep disorder $63.6 \%$ \\
\hline $\begin{array}{l}\text { Zhao et } \\
\text { al., } \\
2020\end{array}$ & $\begin{array}{l}\text { Cross- } \\
\text { section } \\
\text { al }\end{array}$ & $\begin{array}{l}\text { Comm } \\
\text { unity }\end{array}$ & $\begin{array}{l}\text { Mixe } \\
\text { d }\end{array}$ & China & $\begin{array}{l}\text { Chines } \\
\mathrm{e} \\
\text { populat } \\
\text { ion }\end{array}$ & $\begin{array}{l}29.17 \\
\pm \\
10.58\end{array}$ & $\begin{array}{l}\text { Insomnia, } \\
\text { anxiety }\end{array}$ & $\begin{array}{l}\text { Onlin } \\
\mathrm{e}\end{array}$ & PSQI & $\begin{array}{l}\text { PSQ } \\
\text { I } \\
\text { scor } \\
\text { e }>5\end{array}$ & 1630 & & $\begin{array}{c}\text { Not } \\
\text { specified }\end{array}$ & $\begin{array}{l}39.1 \% \text { of frontline workers had } \\
\text { symptoms of insomnia on the ISI scale }\end{array}$ \\
\hline $\begin{array}{l}\text { Zhou et } \\
\text { al., } \\
2020\end{array}$ & $\begin{array}{l}\text { Cross- } \\
\text { section } \\
\text { al }\end{array}$ & $\begin{array}{l}\text { Comm } \\
\text { unity }\end{array}$ & $\begin{array}{l}\text { Schoo } \\
1 \text { and } \\
\text { colleg } \\
\text { e } \\
\text { stude } \\
\text { nts }\end{array}$ & China & $\begin{array}{l}\text { Adoles } \\
\text { cent } \\
\text { and } \\
\text { young } \\
\text { adults }\end{array}$ & $\begin{array}{l}17.41 \\
\pm 2.70\end{array}$ & $\begin{array}{l}\text { Insomnia, } \\
\text { anxiety }\end{array}$ & $\begin{array}{l}\text { Onlin } \\
\mathrm{e}\end{array}$ & PSQI & $\begin{array}{l}\text { PSQ } \\
\text { I } \\
\text { scor } \\
\text { e }>5\end{array}$ & 11835 & $\begin{array}{l}\text { Not } \\
\text { reporte } \\
\text { d }\end{array}$ & $\begin{array}{c}\text { Not } \\
\text { specified }\end{array}$ & $\begin{array}{l}60 \% \text { participants reported of poor sleep } \\
\text { quality. Mean sleep duration was } 5.71 \\
\text { hours (and mean sleep latency was } \\
33.49 \text { minutes (SD=28.87). A total of } \\
76 \%, 81 \%, 45 \% \text {, and } 19 \% \text { reported } \\
\text { difficulty initiating sleep (DIS), } \\
\text { difficulty maintaining sleep (DMS) or } \\
\text { early morning awakening (EMA), } \\
\text { nightmares and using hypnotics } \\
\text { respectively. }\end{array}$ \\
\hline $\begin{array}{l}\text { Zhou et } \\
\text { al., } \\
2020\end{array}$ & $\begin{array}{l}\text { Cross- } \\
\text { section } \\
\text { al }\end{array}$ & $\begin{array}{l}\text { Comm } \\
\text { unity }\end{array}$ & $\begin{array}{l}\text { Not } \\
\text { report } \\
\text { ed }\end{array}$ & $\begin{array}{l}\text { United } \\
\text { States }\end{array}$ & $\begin{array}{l}\text { Quaran } \\
\text { tined } \\
\text { young } \\
\text { adults }\end{array}$ & 25.5 & Insomnia & online & ISI & $\begin{array}{l}\text { Tota } \\
1 \text { ISI } \\
\text { scor } \\
\mathrm{e} \geq 8\end{array}$ & 208 & $36.10 \%$ & $\begin{array}{l}\text { Explorato } \\
\text { ry }\end{array}$ & $\begin{array}{l}\text { Intervention: Baduanjin Exercise twice } \\
\text { daily under guidance of professionals. } \\
\text { Signiant differences found in the } \\
\text { Baduanjin group included a } 43.9 \% \\
\text { lower }(\mathrm{p}<0.001) \text { in the GAD-7 score } \\
\text { and an approximately } 75.9 \% \text { higher } \\
\text { ( } \mathrm{p}=0.003 \text { ) in SMH Sleep Questionnaire } \\
\text { score compare with the control group at } \\
\text { discharge. }\end{array}$ \\
\hline $\begin{array}{l}\text { Chen et } \\
\text { al., } \\
2020\end{array}$ & $\begin{array}{l}\text { Case- } \\
\text { Control }\end{array}$ & $\begin{array}{l}\text { Hospita } \\
1\end{array}$ & $\begin{array}{l}\text { Not } \\
\text { report } \\
\text { ed }\end{array}$ & China & $\begin{array}{l}\text { COVI } \\
\text { D } \\
\text { patients }\end{array}$ & $\begin{array}{r}\text { Case- } \\
57.23 \\
\pm \\
14.37 \\
\text { Contr }\end{array}$ & $\begin{array}{l}\text { Insomnia, } \\
\text { anxiety }\end{array}$ & $\begin{array}{l}\text { face } \\
\text { to } \\
\text { face }\end{array}$ & $\begin{array}{l}\text { SMH } \\
\text { Sleep } \\
\text { Questionn } \\
\text { aire }\end{array}$ & & 78 & $43.6 \%$ & $\begin{array}{l}\text { Not } \\
\text { specified }\end{array}$ & $\begin{array}{l}\text { Intervention: Progressive muscle } \\
\text { relaxation (PMR) technology for } 30 \\
\text { min every day. The average sleep } \\
\text { quality score (SRSS) of the two groups } \\
\text { before intervention was not statistically }\end{array}$ \\
\hline
\end{tabular}




\begin{tabular}{|c|c|c|c|c|c|c|c|c|c|c|c|c|c|c|}
\hline & & & & & & $\begin{array}{r}\text { ol- } \\
60.54 \\
\pm \\
16.34 \\
\end{array}$ & & & & & & & & $\begin{array}{l}\text { significant }(\mathrm{P} 1 / 40.838) \text {, and it was } \\
\text { statistically significant after } \\
\text { intervention }(\mathrm{P}<0.001) \text {. }\end{array}$ \\
\hline $\begin{array}{l}\text { Liu et } \\
\text { al., } \\
2020\end{array}$ & RCT & $\begin{array}{l}\text { Hospita } \\
1\end{array}$ & $\begin{array}{l}\text { Not } \\
\text { report } \\
\text { ed }\end{array}$ & China & $\begin{array}{l}\text { COVI } \\
\text { D } \\
\text { patients }\end{array}$ & $\begin{array}{l}50.41 \\
\pm \\
13.04\end{array}$ & $\begin{array}{l}\text { Insomnia, } \\
\text { anxiety }\end{array}$ & $\begin{array}{l}\text { face } \\
\text { to } \\
\text { face }\end{array}$ & SRSS & & 51 & $56 \%$ & $\begin{array}{l}\text { Random } \\
\text { sampling }\end{array}$ & $\begin{array}{l}\text { The total average PSQI of the } \\
\text { experimental group was } 16.07 \pm 3.761 \text {, } \\
\text { indicating that the sleep quality was } \\
\text { poor. Among them, participants with } \\
\text { moderate insomnia reached } 61.67 \% \text {, } \\
\text { and participants with severe insomnia } \\
\text { reached } 26.67 \% \text {. }\end{array}$ \\
\hline $\begin{array}{l}\text { Wu et } \\
\text { al., } \\
2020\end{array}$ & $\begin{array}{l}\text { Survey } \\
\text { study }\end{array}$ & $\begin{array}{l}\text { Hospita } \\
1\end{array}$ & $\begin{array}{l}\text { Mixe } \\
\text { d }\end{array}$ & China & $\begin{array}{l}\text { Frontli } \\
\text { ne } \\
\text { medica } \\
1 \\
\text { worker } \\
\mathrm{s}\end{array}$ & $\begin{array}{l}\text { Not } \\
\text { report } \\
\text { ed }\end{array}$ & $\begin{array}{l}\text { Somatizat } \\
\text { ion, } \\
\text { depressio } \\
\text { n, anxiety, } \\
\text { insomnia }\end{array}$ & $\begin{array}{l}\text { face } \\
\text { to } \\
\text { face }\end{array}$ & PSQI & $\begin{array}{l}\text { PSQ } \\
\text { I } \\
\text { scor } \\
e>7\end{array}$ & 120 & $26.7 \%$ & $\begin{array}{l}\text { Convenie } \\
\text { nt } \\
\text { sampling }\end{array}$ & \\
\hline
\end{tabular}

*AIS= Athens Insomnia Scale, SPSQI= Short, ISI = Insomnia Severity Index, PSQI= Pittsburgh Sleep Quality index, CPSQI= Chinese Pittsburgh Sleep Quality index, DBAS-16= Dysfunctional Beliefs and Attitudes about Sleep, ESS= Epworth Sleepiness Scale, IPSQI= Italian Pittsburgh Sleep Quality index, CISI = Chinese Insomnia Severity Index, MOSSS = Medical Outcomes Study Sleep Scale, WHIIRS = Women's Health Initiative Insomnia Rating Scale, JSS= Jenkins Sleep Scale , CSHQ = Children's Sleep Habits Questionnaire, SRSS= Sleep State Self-Rating Scale (SRSS) 


\subsection{Characteristics of study population:}

\subsubsection{Education:}

Most studies did not report stratified levels of education among the participants or recruited people with mixed education status. In $16.25 \%(\mathrm{n}=13)$ of studies, the participants had at least bachelor's degree. For example, according to Li et al., ${ }^{48} 88.37 \%$ of the participants had bachelor's degree. Only $20 \%(\mathrm{n}=16)$ of the studies included participants with graduate degree or above. According to Bhargava et al., ${ }^{49}$ all the participants included in the study were specialized doctors who had graduate degrees or above. Furthermore, $26.25 \%(\mathrm{n}=21)$ studies recruited participants with various level of education. For example, Dai et al., ${ }^{50}$ reported that, $60.69 \%$ of the recruited participants had undergraduate degree, $31.99 \%$ had graduate degree and $7.32 \%$ had high school degree.

\subsubsection{Population Specifics:}

Nearly one third $(\mathrm{n}=22,27.5 \%)$ studies were based on healthcare workers. For example, the study by Korkmaz et al., ${ }^{37}$ was conducted among nurses, physician and other health care staff in the hospital. One fourth of the studies $(n=20)$ focused on general population. For example, Innocenti et al., ${ }^{46}$ invited general populations of Italy to participate in their study. Alongside, $8.75 \%(n=7)$ of the studies included only COVID-19 patients. Diomidous et al., ${ }^{39}$ included participants $(n=204)$ from Greece who were confirmed cases of COVID-19. A few studies, $(n=$ $6,7.5 \%$ ) included only quarantined people. For example, Salehinejad et al., ${ }^{51}$ included participants who were quarantined at home during COVID-19.

\subsubsection{Age:}

Mean age ranged from 7.89 years to 63.9 years. Türkoğlu et al., ${ }^{45}$ included 46 children with autism spectrum disorder and the mean age of the participants in this study was 7.89 years. Pinto et al., ${ }^{52}$ a study based on Portugal population, included 365 participants in their study and the mean age of the participants was 63.9 years.

\subsubsection{Gender (\% of males):}

The total percentage of male participants in the study varied widely. It ranged from $3 \%$ to $82 \%$, with a median value of $37.5 \%$. Nevertheless, in majority of the studies the percentage of 
male participants were between 40\%-60\%. For example, Gupta et al., ${ }^{53}$ reported of $58 \%$ male participants whereas, $\mathrm{Li}$ et al., ${ }^{54}$ reported of $43 \%$ male participants.

\subsection{Prevalence of sleep disorder:}

The prevalence of sleep disorders varied across the samples. Ranging from $2.3 \%$ to $76.6 \%$ For example, Zhang et al., ${ }^{55}$ reported the overall prevalence of insomnia was as high as $76.6 \%$ among the medically isolated population. The prevalence rates varied across geographic regions of corresponding primary studies. For example, the prevalence of insomnia in the United States ranged between $20-41 \%$. For example, $30 \%$ participants reported of some degree of insomnia in the study by Bhargava et al., ${ }^{49}$ in the USA. Whereas, a significant variation was noted in the prevalence of insomnia in Chinese population. Although only $2.3 \%$ of the Chinese workforce reported of insomnia ${ }^{56}, 58 \%$ of the frontline medical staff were suffering from insomnia ${ }^{44}$. Such variation was also noted in Italy, where the prevalence of insomnia ranged from $37.6 \%$ to $57 \%$. For example, according to Casagrande et al., ${ }^{57} 57.1 \%$ participants in Italy had insomnia and poor sleep quality.

\subsection{Factors associated with sleep disorders during COVID-19:}

\subsubsection{Sex:}

$20 \%$ of the studies $(\mathrm{n}=15)$ reported that females had a higher risk of some degree of insomnia. For example, Batool-Anwar et al., ${ }^{58}$ reported that stratification by gender revealed worsening insomnia only among women. However, two studies also reported that males had higher risk of insomnia. For example, male participants in the study by Anzar et al., ${ }^{59}$ reported higher prevalence of insomnia and poor sleep quality.

\subsubsection{Age:}

Only 7 studies reported that younger population had worse sleep compared to their older counterpart. For example. Beck et al., ${ }^{60}$ mentioned that, younger population reported higher sleep disturbance compared to older population (79\% vs 72\%). Nevertheless, 4 studies reported of poor sleep among the older population as well. For example, Wang et al., ${ }^{61}$ reported that older population had 1.42 times higher risk of insomnia.

\subsubsection{Education:}


Several studies found that level of education was associated with worse sleep. Zhang et al., ${ }^{62}$ reported that education level of high school or below increased the risk of insomnia but ${ }^{33}$ reported that participants having higher educational level had worse sleep.

\subsubsection{Social support:}

Social support was an important factor associated with sleep. Four studies reported that those with lack of social support had higher prevalence of insomnia and poor quality of sleep. For example, Tselebi et al., ${ }^{47}$ reported participants with lack of family support had higher level of insomnia. Individuals in isolation ${ }^{52}$, living alone ${ }^{63}$ suffering from loneliness ${ }^{64}$ or being single ${ }^{44}$ also reported of high degree of insomnia. This further emphasizes the impact of social support on sleep.

\subsubsection{Physical health:}

Individuals with preexisting chronic disease $(n=4)$ and perceived poor health $(n=5)$ suffered from higher prevalence of insomnia.

\subsubsection{Mental health:}

Insomnia worsened among patients with preexisting psychiatric illness $(n=4)$. Stress $(n=$ 6), anxiety $(\mathrm{n}=8)$, depression $(\mathrm{n}=4)$, fatigue Zhang et al., ${ }^{62}$ and low mood Carrigan et al., ${ }^{65}$ was also found to be associated with poor sleep.

\subsubsection{Lack of Physical activity:}

Three studies reported that reduced opportunities of physical activities were associated with poor sleep among participants.

\subsubsection{Being Health Care Worker:}

Nearly $13 \%(\mathrm{n}=10)$ of the studies reported that, health care workers were at risk of some degree of insomnia. For example, Anzar et al., ${ }^{59}$ reported that HCW had not only a higher prevalence of insomnia but also had reduced quality of sleep.

Four studies reported that the health care workers at risk of contracting COVID-19 had poor sleep quality. For example, Carrigan et al., ${ }^{65}$ found that, being at risk of CVID-19 at work lowers the quality of sleep. Zhang et al., ${ }^{62}$ also found that working in the isolation unit impaired 
the sleep cycle among HCWs. Frontline medical workers suffered from higher level of insomnia compared to non-frontline workers. For example, Qi et al., ${ }^{66}$ reported that Frontline worker in the Wuhan, China (epicenter of the outbreak in China) scored significantly higher in the PSQI scale compared to non-front liners in Ningbo. Shift work ${ }^{28}$, reluctance to join the frontline ${ }^{67}$ and higher burden of work ${ }^{68}$ also played an important role in sleeping.

\subsubsection{Factors Specific to COVID-19:}

Two studies reported that patients suffering from COVID-19 reported poor quality of sleep. Fear of COVID-19 $(n=2)$ and worry about the disease $(n=5)$ also affected the quality of sleep. Uncertainty due to the pandemic $(n=5)$, negative attitude toward control measures such as wearing mask ${ }^{69}$ and lack of availability of testing ${ }^{50}$ were the other factors specific to COVID-19 associated with sleep.

\subsubsection{Factors related to work:}

Several factors related to employment also impacted sleep significantly. One study found that people with low income had higher risk of sleep disorder compared to their high-income counterparts $^{33}$. Worrying about impact of this pandemic on livelihood, possible negative impact on their income was also associated with sleep disorders ${ }^{63}$. One study mentioned that being at home without any work also impaired regular sleep cycle ${ }^{52}$. On the contrary, stress for returning to work after the lockdown did not impair sleep ${ }^{56}$.

\subsection{Interventions for sleep disorders:}

There were only two interventions for sleep disorders. The study by Chen et al., ${ }^{70}$ was conducted among COVID-2019 patients, where the intervention group performed Baduanjin exercise (traditional Chinese mind-body exercise routine) under professional guidance and the controls received usual care. Their anxiety and insomnia level was measured using GAD-7 and SMH Sleep questionnaire at baseline and discharge. Although there was no significant difference among these groups at baseline, intervention group showed $44 \%$ lower score $(\mathrm{P}<0.001)$ in GAD and $76 \%$ lower score $(\mathrm{P}=0.003)$ in the $\mathrm{SMH}$ Sleep questionnaire at discharge which indicated that Baduanjin exercise can be beneficial for improving anxiety and insomnia among COVID-19 patients. 
In the randomized controlled clinical trial by Liu et al., ${ }^{36}$ patients with COVID-19 patients who entered the isolation ward were randomly divided into experimental and control groups where the experimental group used progressive muscle relaxation (PMR) technology for 30 min per day for 5 consecutive days and the control group received only routine care and treatment. The Spielberger State-Trait Anxiety Scale (STAI) and Sleep State Self-Rating Scale (SRSS) were used to measure and record patient anxiety and sleep quality pre and post intervention. Both the group had comparable scores before intervention however, STAI and SRSS scores reduces significantly after intervention $(\mathrm{P}<0.001)$.

\section{Discussion:}

\subsection{Overview of the synthesized findings}

After a thorough review of the available evidence, there were 78 studies that met the pre-set criteria of this scoping review. Most of the studies included were cross-sectional in design, and online questionnaires were the common mode of data collection. Studies were conducted both in the community and hospital settings. Most of the studies on sleep disorders were conducted in China or the USA. Insomnia, anxiety, and depression were commonly assessed disorders, and PSQI, ISIS, and AIS were used to evaluate insomnia and sleep quality. The findings from these existing literatures inform a high burden of sleep disorder during this pandemic. The prevalence of sleep disorder varied widely across the samples and ranged from $2.3 \%-76.6 \%$. The majority of the studies reported females had a higher risk of some degree of insomnia. Although older adults generally have a higher rate of insomnia, many studies have reported that younger generations were having difficulties with sleeping during the pandemic. Covariates-adjusted research was limited which could explain how age played a role in sleep patterns among the study samples. Moreover, the education level was also associated with sleep disorders, but there was ambiguity regarding the specific educational status. Both higher education and high school level education were found to impact sleep. This can be attributable to the fact the educated individuals or students may have academic and professional stressors that may have impacted their mental health and sleep conditions ${ }^{71-73}$. Furthermore, social support was found to have a critical role in sleep status and associated disorders. Lack of social support, family support, living alone, and isolation was associated with a higher risk of sleep disorders. 
This review also found that comorbid physical and mental illness increased the risks of insomnia. Health care workers, especially those serving the frontline, were at high risk of insomnia during the COVID-19 pandemic. Increased workload, shift work, fear of contracting the virus were the significant risk factors among the $\mathrm{HCW}$. This might have resulted in higher psychosocial stress and burnout, which can be associated with sleep disorders among the frontlines ${ }^{74}$. The fear of contracting the virus, worry about the disease, uncertainty regarding treatment and prevention measures, and negative attitude toward control measures were COVID-19 specific risk factors for sleep disorders. Other related factors were lack of opportunity for physical activity, fear of the negative impact on income, and staying at home without employment. Only two interventions were identified, which focused either on baduanjin exercise (as one of the traditional Chinese mind-body exercise) or progressive muscular reaction. Both reported significant improvement on sleep score after the intervention ${ }^{75,76}$

The findings of this current review are comparable with the previous literature. Our review reports a considerably higher prevalence of both insomnia and insomnia symptoms higher during this pandemic. Several reviews have also reported higher prevalence after stressful events like stroke, chronic injuries ${ }^{77}$. The current review reports that females were at higher risk on insomnia and many studies have found that female are at high risk several mental illnesses such as anxiety and depression ${ }^{78}$.

Despite the lack of accurate normative data on evolution of sleep architecture with age, several studies suggest that sleep patterns of the individuals change across the lifespan. In older adults have a significant delay in sleep latency and decrease in total REM sleep ${ }^{79}$. These findings suggest that older adults have more problems with sleep compared to the younger population. However, several studies included in this review reported that younger population had more trouble with sleep. This could be explained by the preexisting and emerging academic and processional uncertainty and the mental stressors amid this pandemic.

Our findings are also in line with evidence suggesting that individuals with insomnia have a higher risk of psychiatric disorders and sleep disturbances may facilitate the onset of several mental health illness. Many studies have reported that there is a bidirectional relationship between anxiety, depression and insomnia ${ }^{80}$. In this circumstance high rate of insomnia among individuals with preexisting psychological was unsurprising. 
Our review shed light on the high prevalence of insomnia and sleep disturbances among health care workers especially those in frontline. Studies from previous major outbreaks of SARS, MERS have also reported of elevation of psychological distress among HCW during and after the outbreak which interfered with their social and occupational functioning ${ }^{81-83}$. We need to learn from the history and intervene at the early stage to support these HCWs.

\section{Limitations of the review}

This scoping review has several limitations. The foremost limitation of the current literature is that most studies are cross-sectional or longitudinal design, most of which did not collect data before the onset of the pandemic to provide a comparative picture of the situation. The limited number of studies reporting prevalence at multiple time points made it difficult to determine how the insomnia status of individuals changed over time.

To investigate temporality and establish causal relationships, risk factors should be captured before the inception of the disease. Owing to this, causal relationship was not established. Therefore, the conclusion of this scoping review is limited to demonstrating association only.

Additionally, there is a risk of potential selection bias as we did not search all the databases and excluded unpublished studies, reports, and studies in languages other than English. Moreover, there could be a publication bias within the scientific literature as less significant findings are less likely to get published, thus may not contribute to the evidence base. Furthermore, we did not conduct a quantitative evaluation of the patient-level data, which could have eliminated withinstudy and between-study variations to provide uniform prevalence estimations across samples.

Another notable limitation of this review was limiting the search strategy within bibliometric databases, whereas a major proportion of the literature can be available on preprint servers. As we aimed to synthesize the peer-reviewed empirical research, we did not search the preprint sources that might have had early stage findings rather than peer-reviewed articles. Lastly, as most studies were conducted in China and the USA, our findings' generalizability may be limited because of the wide variation of cultural norms and the healthcare system across the globe. Sleep disorders and co-morbid mental health problems may be highly prevalent in people living in low-income countries that are underrepresented in the existing studies ${ }^{84,85}$. Also, people with preexisting neuropsychiatric conditions or psychosocial vulnerabilities may experience a 
disproportionate burden of sleep disorders, that is yet to be examined through empirical research. Our review may not have captured the epidemiology of sleep disorders in those marginalized populations.

\subsection{Implications for future research}

The evidence landscape on COVID-19 and associated mental health problems has been changing rapidly ${ }^{86,87}$. This scoping review examined the epidemiological aspects of sleep disorders. As this review includes literature up to August 12, 2020, the synthesized evidence can be different from those studies published after that date, or those research that are being conducted now. However, as the goal of a scoping review is to chart the initial evidence in a scientific topic, the findings if this review can serve as the basis of future research focusing on specific subtopics of sleep disorders amid this pandemic. Such areas can be examined using specific methods appropriate for respective research objectives.

As most studies in this review were cross-sectional in nature, future studies should adopt longitudinal designs that may explore how the magnitude and correlators of sleep disorders evolve over time. Moreover, it is essential to examine the comorbid physical and mental health problems that are associated with sleep disorders (Hossain et al., 2020). Understanding the syndemic effects of multiple health problems may better inform why the prevalence and risk factors of sleep disorders may vary across samples ${ }^{88,89}$. Furthermore, it is essential to examine the mental health problems associated with sleep disorders in high risk populations such as those with neurodevelopmental disorders or social-economically marginalized populations. Evaluating the Epidemiology of sleep disorders in diverse population groups through cohort studies may provide valuable insights not only during this pandemic but also how different populations may experience unique psychosocial challenges during public health emergencies.

One of the notable findings of this review was the use of multiple scales for assessing sleep disorders as well as associated mental health problems. Many scholars have adopted previous scales, where is several new scales were introduced during this pandemic ${ }^{90}$. It is essential to conduct extensive factor analysis evaluating the appropriateness of multiple skills so that future research can be conducted using standardized scales and measurements. such efforts may provide a more accurate scenario of the epidemiology of sleep disorders. 
The emergence of online data collection measures may provide easy access to people who may use those platforms; however, people with digital divide may not participate in such studies $^{91,92}$. it is essential to consider such limitations of the existing research efforts and design inclusive methodology's that may include diverse populations. In future research, measurements on psychosocial health outcomes including sleep disorders can be included in primary care and hospital health records that may offer valuable information on sleep disorders and other mental health problems. Nonetheless, Population based research exploring health and social aspects of COVID-19 may include instruments measuring the impacts of this pandemic on sleep behavior in different populations. Such approaches may improve the research production and the quality of data on Epidemiology of sleep disorders during this pandemic.

\subsection{Implications for mental health policymaking and practice}

This review examined the Epidemiology of sleep disorders, which may provide meaningful insights for mental health policy making and practice. The ongoing interventions and preventive measures adopted in different contexts should revisit the components that may influence sleep outcomes across populations. Institutional and local policies and programs on mental health should not only target mental disorders such as anxiety and depression, but also emphasize on sleep disorders that are highly prevalent in COVID-19 affected populations. Specific provisions should be made to prevent, diagnose, and treat sleep disorders. The emergence of telemedicine and online support systems provide unique opportunities from a public mental health perspective ${ }^{93}$. such services should be developed and implemented considering the burden and risk factors of sleep disorders during this pandemic. psychiatrists and psychologists may access over arcing risk and protective factors of sleep disorders and other mental health problems that can be managed using cost effective and evidence-based approaches. It is necessary to adopt such guidelines and services in psychosocial care policies and programs in different contexts. such measures can be customized using local mental health data considering the available mental health resources in those contexts. Furthermore, pandemic preparedness and mental health action plans should be informed by the epidemiological evidence on sleep disorders that may potentially impact the health and well being of health care providers, hospitalized patients, informal caregivers, and population at risk during COVID-19 pandemic. 
Evidence-based pharmacological and psychosocial interventions have paramount importance for addressing sleep disorders. However, despite the elaborate searching through the databases, only two interventions were identified that focused on improving insomnia during the pandemic. Both of these interventions took place inside hospitals among patients admitted with COVID-19. Whereas, our review indicates a high prevalence of insomnia among patients, HCW as well as the general population. This necessitates community-based interventions to improve sleep among the target population. Mental health practitioners may access the relevance and appropriateness of existing interventions that may help during this pandemic. It is essential to conduct implementation research examining the effectiveness and efficiency of available interventions and develop newer interventions that may target specific psychosocial stressors for sleep disorders during this pandemic.

The findings of this review, emphasizes the need for early detection and effective treatment all the symptoms of insomnia, including the mild ones, before they evolve to more complex and evokes enduring psychological responses. Current knowledge of prevalence, type and comorbidities of prevalence, symptom profiles and comorbidities of insomnia should be utilized and tailored interventions targeting the behavioral components should be further developed to address the issue.

It has been evident that there is widespread of insomnia among the general population in this pandemic. All patients coming in contact with health care facilities should be screened, and those diagnosed with any form of insomnia should be referred to appropriate resources. As healthcare workers reported a higher burden of insomnia, specific interventions designed for addressing the problems at health care facilities should be available. The majority of health care workers are overburdened. If their optimal health and wellness are not ensured, their ability to work will be further compromised impose a severe predicament on the current fragile healthcare system.

Cognitive behavioral therapy for insomnia (CBT-I) is the treatment of choice for insomnia. A systematic review and meta-analysis of RCTs reported that, CBI-I is the effective mode of treatment for insomnia capable of producing clinically meaningful effect size with no identifiable adverse effects ${ }^{94}$. Several studies have also shown small to large effects on efficiency and quality and sleep onset latency along with reduction in severity of insomnia, wake after sleep onset and 
number of awakenings ${ }^{95}$. Moreover, a full economic evaluation of CBT-I in adult populations revealed CBT-I was cost-effective compared to pharmacotherapy or no treatment. Thus, incorporation of some form of CBT-I in clinical practice will improve the overall sleep status of the population.

If the delivery of in-person CBT-I is not possible due to the social distancing measures for preventing the spread of the virus, similar interventions can be delivered using the virtual platforms. CBT-I delivered though internet or computer was also found to be as effective and a viable alternative in the current context ${ }^{96-98}$. Alongside, other avenues of telepsychiatry such as virtual clinics, remotely delivered psychotherapies, psychoeducation, 24/7 chat lines and digital monitoring should also be explored.

For areas with poor internet connection or individuals who are not accustomed with modern intervention formats, self-help books may be an effective alternative which could possibly reduce ensure standardization of care, quality control and optimal resource utilization ${ }^{99}$.

Accumulating evidence show that exercise and dietary interventions are associated with improved quality of sleep. A large RCT conducted in China reported that low fat diet and increased amount of exercise resulted in improved sleep by altering the metabolic pathways. Future intervention research should focus on how lifestyle-based interventions can impact sleep quality and overall mental health in different populations at risk. It is critical to assess the risk and protective factors that can be used to develop mental health promotion programs that potentially prevent sleep disorders among the vulnerable individuals. The scope of digital interventions, peer support groups community-based mental health services, self-management programs, And public mental health resources should be evaluated for promoting mental health and preventing sleep disorders across populations.

\section{Conclusions:}

Amid this global pandemic burden of mental health issues are becoming a growing concern in addition to infection control. Sleep disorders are significant mental health problems associated with increased psychosocial stressors. Findings from this review suggest a high burden of sleep disorder across different population groups. Female gender, younger population, HCWs, COVID19 related stressors were the major factors associated with sleep disorder identified in this review. 
Despite the high burden, a limited number of interventions were identified to address this problem. Early diagnosis of sleep disorder and adequate treatment is crucial to prevent further worsening of the condition. Evidence-based pharmacological and psychosocial interventions have paramount importance for addressing sleep disorders. Future studies should explore interventions that utilize digital platforms and adopt innovative strategies in order to ensure increased outreach and sustainability.

\section{References:}

1. Qu G, Li X, Hu L, Jiang G. An Imperative Need for Research on the Role of Environmental Factors in Transmission of Novel Coronavirus (COVID-19). Environ Sci Technol. 2020. doi:10.1021/acs.est.0c01102

2. Sharma VK, Jinadatha C, Lichtfouse E. Environmental chemistry is most relevant to study coronavirus pandemics. Environ Chem Lett. 2020. doi:10.1007/s10311-020-01017-6

3. WHO Coronavirus Disease COVID-19 Dashboard. 2020. https://covid19.who.int/.

4. Torales J, O'Higgins M, Castaldelli-Maia JM, Ventriglio A. The outbreak of COVID-19 coronavirus and its impact on global mental health. Int J Soc Psychiatry. 2020. doi:10.1177/0020764020915212

5. Rossi R, Socci V, Talevi D, et al. COVID-19 Pandemic and Lockdown Measures Impact on Mental Health Among the General Population in Italy. Front Psychiatry. 2020. doi:10.3389/fpsyt.2020.00790

6. Hossain MM, Sultana A, Purihit N. Mental health outcomes of quarantine and isolation for infection prevention: A systematic umbrella review of the global evidence. 2020. https://doi.org/10.31234/osf.io/dz5v2.

7. DiGiovanni C, Conley J, Chiu D, Zaborski J. Factors influencing compliance with quarantine in Toronto during the 2003 SARS outbreak. Biosecur Bioterror. 2004. doi:10.1089/bsp.2004.2.265

8. Hawryluck L, Gold WL, Robinson S, Pogorski S, Galea S, Styra R. SARS control and 
psychological effects of quarantine, Toronto, Canada. Emerg Infect Dis. 2004. doi:10.3201/eid1007.030703

9. Wu P, Fang Y, Guan Z, et al. The psychological impact of the SARS epidemic on hospital employees in China: Exposure, risk perception, and altruistic acceptance of risk. Can J Psychiatry. 2009. doi:10.1177/070674370905400504

10. Bai YM, Lin CC, Lin CY, Chen JY, Chue CM, Chou P. Survey of stress reactions among health care workers involved with the SARS outbreak. Psychiatr Serv. 2004. doi:10.1176/appi.ps.55.9.1055

11. Brooks SK, Webster RK, Smith LE, et al. The psychological impact of quarantine and how to reduce it: rapid review of the evidence. Lancet. 2020. doi:10.1016/S01406736(20)30460-8

12. Krystal AD. Psychiatric Disorders and Sleep. Neurol Clin. 2012. doi:10.1016/j.ncl.2012.08.018

13. Staner L. Sleep and anxiety disorders. Dialogues Clin Neurosci. 2003.

14. Otsuka Y, Kaneita Y, Itani O, Nakagome S, Jike M, Ohida T. Relationship between stress coping and sleep disorders among the general Japanese population: a nationwide representative survey. Sleep Med. 2017. doi:10.1016/j.sleep.2017.06.007

15. Colten HR, Altevogt BM. Sleep Disorders and Sleep Deprivation: An Unmet Public Health Problem.; 2006. doi:10.17226/11617

16. Seng EK, Cervoni C, Lawson JL, et al. The burden of sleep problems: A pilot observational study in an ethnically diverse urban primary care setting. J Prim Care Community Heal. 2016. doi:10.1177/2150131916651068

17. Chattu V, Manzar M, Kumary S, Burman D, Spence D, Pandi-Perumal S. The Global Problem of Insufficient Sleep and Its Serious Public Health Implications. Healthcare. 2018. doi:10.3390/healthcare7010001

18. Hillman DR, Lack LC. Public health implications of sleep loss: The community burden. Med J Aust. 2013. doi:10.5694/mja13.10620 
19. Rajkumar RP. COVID-19 and mental health: A review of the existing literature. Asian J Psychiatr. 2020. doi:10.1016/j.ajp.2020.102066

20. Peters MDJ, Godfrey CM, Khalil H, McInerney P, Parker D, Soares CB. Guidance for conducting systematic scoping reviews. Int J Evid Based Healthc. 2015;13(3):141-146. doi:10.1097/XEB.0000000000000050

21. Tricco AC, Lillie E, Zarin W, et al. PRISMA Extension for Scoping Reviews (PRISMAScR): Checklist and Explanation. Ann Intern Med. 2018;169(7):467. doi:10.7326/M180850

22. Tasnim S, Rahman M, Pawar P, et al. Epidemiology of sleep disorders during COVID-19 pandemic: A systematic scoping review protocol | OSF Registries. OSF Regist. 2020. doi:10.17605/OSF.IO/J67DG

23. World Health Organization. WHO | ICD-10 online versions. WHO. 2010.

24. American Psychiatric Association. Diagnostic and Statistical Manual of Mental Disorders.

25. Thorpy MJ. Classification of Sleep Disorders. Neurotherapeutics. 2012;9(4):687-701. doi:10.1007/s13311-012-0145-6

26. Ouzzani M, Hammady H, Fedorowicz Z, Elmagarmid A. Rayyan-a web and mobile app for systematic reviews. Syst Rev. 2016. doi:10.1186/s13643-016-0384-4

27. Nalleballe K, Reddy Onteddu S, Sharma R, et al. Spectrum of neuropsychiatric manifestations in COVID-19. Brain Behav Immun. 2020. doi:10.1016/j.bbi.2020.06.020

28. Gao C, Scullin MK. Sleep health early in the coronavirus disease 2019 (COVID-19) outbreak in the United States: integrating longitudinal, cross-sectional, and retrospective recall data. Sleep Med. 2020. doi:10.1016/j.sleep.2020.06.032

29. Amerio A, Bianchi D, Santi F, et al. Covid-19 pandemic impact on mental health: A webbased cross-sectional survey on a sample of Italian general practitioners. Acta Biomed. 2020. doi:10.23750/abm.v91i2.9619

30. Voitsidis P, Gliatas I, Bairachtari V, et al. Insomnia during the COVID-19 pandemic in a Greek population. Psychiatry Res. 2020. doi:10.1016/j.psychres.2020.113076 
31. Xiao H, Zhang Y, Kong D, Li S, Yang N. The effects of social support on sleep quality of medical staff treating patients with coronavirus disease 2019(COVID-19) in January and February 2020 in China. Med Sci Monit. 2020. doi:10.12659/MSM.923549

32. Renzo L Di, Gualtieri P, Cinelli G, et al. Psychological aspects and eating habits during covid-19 home confinement: Results of ehlc-covid-19 italian online survey. Nutrients. 2020. doi:10.3390/nu12072152

33. Fu W, Wang C, Zou L, et al. Psychological health, sleep quality, and coping styles to stress facing the COVID-19 in Wuhan, China. Transl Psychiatry. 2020. doi:10.1038/s41398-020-00913-3

34. Wright KP, Linton SK, Withrow D, et al. Sleep in university students prior to and during COVID-19 Stay-at-Home orders. Curr Biol. 2020. doi:10.1016/j.cub.2020.06.022

35. Gualano MR, Lo Moro G, Voglino G, Bert F, Siliquini R. Effects of COVID-19 lockdown on mental health and sleep disturbances in Italy. Int J Environ Res Public Health. 2020. doi:10.3390/ijerph17134779

36. Liu K, Chen Y, Wu D, Lin R, Wang Z, Pan L. Effects of progressive muscle relaxation on anxiety and sleep quality in patients with COVID-19. Complement Ther Clin Pract. 2020. doi:10.1016/j.ctcp.2020.101132

37. Korkmaz S, Kazgan A, Çekiç S, Tartar AS, Balcı HN, Atmaca M. The anxiety levels, quality of sleep and life and problem-solving skills in healthcare workers employed in COVID-19 services. J Clin Neurosci. 2020. doi:10.1016/j.jocn.2020.07.073

38. Zhou SJ, Wang LL, Yang R, et al. Sleep problems among Chinese adolescents and young adults during the coronavirus-2019 pandemic. Sleep Med. 2020.

doi:10.1016/j.sleep.2020.06.001

39. Diomidous M. Sleep and Motion Disorders of Physicians and Nurses Working in Hospitals Facing the Pandemic of COVID 19. Med Arch (Sarajevo, Bosnia Herzegovina). 2020. doi:10.5455/medarh.2020.74.210-215

40. Abdulah DM, Musa DH. Insomnia and stress of physicians during COVID-19 outbreak. Sleep Med X. 2020. doi:10.1016/j.sleepx.2020.100017 
41. Mazza MG, De Lorenzo R, Conte C, et al. Anxiety and depression in COVID-19 survivors: Role of inflammatory and clinical predictors. Brain Behav Immun. 2020. doi:10.1016/j.bbi.2020.07.037

42. Huang Y, Zhao N. Generalized anxiety disorder, depressive symptoms and sleep quality during COVID-19 outbreak in China: a web-based cross-sectional survey. Psychiatry Res. 2020. doi:10.1016/j.psychres.2020.112954

43. W.D.S. K, S.A. C, E.C. T, F. F, M.A. G, N.S. D. Suicidal ideation during the COVID-19 pandemic: The role of insomnia. Psychiatry Res. 2020.

doi:10.1016/j.psychres.2020.113134 LK http://QT8BH6HW4W.search.serialssolutions.com?sid=EMBASE\&issn=18727123\&id=d oi:10.1016\%2Fj.psychres.2020.113134\&atitle=Suicidal+ideation+during+the+COVID19+pandemic\%3A+The+role+of+insomnia\&stitle=Psychiatry+Res.\&title=Psychiatry+Re search $\&$ volume $=290 \&$ issue $=\&$ spage $=\&$ epage $=\&$ aulast $=$ Killgore $\&$ aufirst $=$ William + D.. . $\&$ auinit=W.D.S.\&aufull=Killgore+W.D.S.\&coden=PSRSD\&isbn=\&pages $=-$ \&date $=2020 \&$ auinit $1=W \&$ auinitm $=$ D.S.

44. J L, S M, Y W, et al. Factors Associated With Mental Health Outcomes Among Health Care Workers Exposed to Coronavirus Disease 2019. JAMA Netw open. 2020.

45. Türkoğlu S, Uçar HN, Çetin FH, Güler HA, Tezcan ME. The relationship between chronotype, sleep, and autism symptom severity in children with ASD in COVID-19 home confinement period. Chronobiol Int. 2020. doi:10.1080/07420528.2020.1792485

46. Innocenti P, Puzella A, Mogavero MP, Bruni O, Ferri R. Letter to editor: CoVID-19 pandemic and sleep disorders - a web survey in Italy. Neurol Sci. 2020. doi:10.1007/s10072-020-04523-1

47. Tselebis A, Sikaras C. Sleep Disorders, Perceived Stress and Family Support Among Nursing Staff During the Pandemic Crisis. :1-13.

48. Li X, Yu H, Bian G, et al. Prevalence, risk factors, and clinical correlates of insomnia in volunteer and at home medical staff during the COVID-19. Brain Behav Immun. 2020. doi:10.1016/j.bbi.2020.05.008 
49. Bhargava S, Sarkar R, Kroumpouzos G. Mental distress in dermatologists during COVID19 pandemic: Assessment and risk factors in a global, cross-sectional study. Dermatol Ther. 2020. doi:10.1111/dth.14161

50. Dai H, Zhang SX, Looi KH, Su R, Li J. Perception of health conditions and test availability as predictors of adults' mental health during the covid-19 pandemic: A survey study of adults in Malaysia. Int J Environ Res Public Health. 2020. doi:10.3390/ijerph17155498

51. Salehinejad MA, Majidinezhad M, Ghanavati E, et al. Negative impact of COVID-19 pandemic on sleep quantitative parameters, quality, and circadian alignment: Implications for health and psychological well-being. EXCLI J. 2020. doi:10.17179/excli2020-2831

52. Pinto J, van Zeller M, Amorim P, et al. Sleep quality in times of Covid-19 pandemic. Sleep Med. 2020. doi:10.1016/j.sleep.2020.07.012

53. Gupta R, Grover S. Changes in sleep pattern and sleep quality during COVID-19 lockdown. 2020.

54. Li DJ, Ko NY, Chen YL, et al. Covid-19-related factors associated with sleep disturbance and suicidal thoughts among the taiwanese public: A facebook survey. Int J Environ Res Public Health. 2020. doi:10.3390/ijerph17124479

55. Zhang WR, Wang K, Yin L, et al. Mental Health and Psychosocial Problems of Medical Health Workers during the COVID-19 Epidemic in China. Psychother Psychosom. 2020. doi:10.1159/000507639

56. Tan W, Hao F, McIntyre RS, et al. Is returning to work during the COVID-19 pandemic stressful? A study on immediate mental health status and psychoneuroimmunity prevention measures of Chinese workforce. Brain Behav Immun. 2020. doi:10.1016/j.bbi.2020.04.055

57. Casagrande M, Favieri F, Tambelli R, Forte G. The enemy who sealed the world: effects quarantine due to the COVID-19 on sleep quality, anxiety, and psychological distress in the Italian population. Sleep Med. 2020. doi:10.1016/j.sleep.2020.05.011

58. Batool-Anwar S, Omobomi OS, Quan SF. Impact of the novel coronavirus disease 
(COVID-19) on treatment adherence and sleep duration in patients with obstructive sleep apnea treated with positive airway pressure. J Clin Sleep Med. 2020.

doi: $10.5664 /$ jcsm. 8746

59. Anzar W, Ali Baig Q, Afaq A, Bin Taheer T, Amar Abstract S. Impact of Infodemics on Generalized Anxiety Disorder, Sleep Quality and Depressive Symptoms among Pakistani Social Media Users during Epidemics of COVID-19*1. Merit Res J Med Med Sci. 2020. doi:10.5281/zenodo.3727246

60. Beck F, Léger D, Fressard L, Peretti-Watel P, Verger P. Covid-19 health crisis and lockdown associated with high level of sleep complaints and hypnotic uptake at the population level. J Sleep Res. 2020. doi:10.1111/jsr.13119

61. Wang J, Gong Y, Chen Z, et al. Sleep disturbances among Chinese residents during the Coronavirus Disease 2019 outbreak and associated factors. Sleep Med. 2020.

doi:10.1016/j.sleep.2020.08.002

62. Zhang C, Yang L, Liu S, et al. Survey of Insomnia and Related Social Psychological Factors Among Medical Staff Involved in the 2019 Novel Coronavirus Disease Outbreak. Front Psychiatry. 2020. doi:10.3389/fpsyt.2020.00306

63. Gaur K, Keshri K, Sharma A, Pachori H. A study of depression , anxiety and insomnia during COVID-19 lockdown in A study of depression, anxiety and insomnia during COVID-19 lockdown in India. 2020;(July).

64. Kokou-Kpolou CK, Megalakaki O, Laimou D, Kousouri M. Insomnia during COVID-19 pandemic and lockdown: Prevalence, severity, and associated risk factors in French population. Psychiatry Res. 2020. doi:10.1016/j.psychres.2020.113128

65. Carrigan N, Wearn A, Meky S, et al. Sleep quality, mental health and circadian rhythms during COVID lockdown: Results from the SleepQuest Study. 2020;44(0). doi:10.1101/2020.07.08.20148171

66. Qi J, Xu J, Li BZ, et al. The evaluation of sleep disturbances for Chinese frontline medical workers under the outbreak of COVID-19. Sleep Med. 2020.

doi:10.1016/j.sleep.2020.05.023 
67. Que J, Shi L, Deng J, et al. Psychological impact of the covid-19 pandemic on healthcare workers: A cross-sectional study in China. Gen Psychiatry. 2020. doi:10.1136/gpsych2020-100259

68. Wang H, Huang D, Huang H, et al. The Psychological Impact of COVID-19 Pandemic on Medical Staff in Guangdong, China: A Cross-Sectional Study. Psychol Med. 2020. doi:10.1017/S0033291720002561

69. Parta C Ramesh, Biswajit K, Parul B. Mental health, sleep quality and quality of life in individuals with and without multiple health conditions during home quarantine in India due to the COVID-19 pandemic: a cross-sectional study. doi:https://doi.org/10.12688/f1000research.24321.1

70. Ming-Gui C, Yunlong Q, Haizhen C, Erthui C. Baduanjin Exercise May Improve the Anxiety and Insomnia in COVID-2019 Patients: A Case-Control Study. 2020. doi:10.21203/rs.3.rs-42755/v1

71. Chi X, Becker B, Yu Q, et al. Persistence and remission of depressive symptoms and psycho-social correlates in Chinese early adolescents. BMC Psychiatry. 2020. doi:10.1186/s12888-020-02808-5

72. Chi X, Becker B, Yu Q, et al. Prevalence and Psychosocial Correlates of Mental Health Outcomes Among Chinese College Students During the Coronavirus Disease (COVID19) Pandemic. Front Psychiatry. 2020. doi:10.3389/fpsyt.2020.00803

73. Chi, Xinli, Chen, Yuying, Chen, Danying chen. Psychometric Evaluation of The Fear of COVID-19 ScaleAmong Chinese Population. 2020.

74. Hossain, Md Mahhub, Tasnim, Samia, Sultana A. Epidemiology of mental health problems in COVID-19: a review. 2020.

75. Zou L, Pan Z, Yeung A, et al. A Review Study on the Beneficial Effects of Baduanjin. $J$ Altern Complement Med. 2018. doi:10.1089/acm.2017.0241

76. Zou L, Yeung A, Quan X, et al. Mindfulness-based baduanjin exercise for depression and anxiety in people with physical or mental illnesses: A systematic review and metaanalysis. Int J Environ Res Public Health. 2018. doi:10.3390/ijerph15020321 
77. Baylan S, Griffiths S, Grant N, Broomfield NM, Evans JJ, Gardani M. Incidence and prevalence of post-stroke insomnia: A systematic review and meta-analysis. Sleep Med Rev. 2020. doi:10.1016/j.smrv.2019.101222

78. Albert PR. Why is depression more prevalent in women? J Psychiatry Neurosci. 2015. doi:10.1503/jpn.150205

79. Roth T, Coulouvrat C, Hajak G, et al. Prevalence and perceived health associated with insomnia based on DSM-IV-TR; international statistical classification of diseases and related health problems, tenth revision; and research diagnostic criteria/international classification of sleep disorders. Biol Psychiatry. 2011. doi:10.1016/j.biopsych.2010.10.023

80. Alvaro PK, Roberts RM, Harris JK. A systematic review assessing bidirectionality between sleep disturbances, anxiety, and depression. Sleep. 2013. doi:10.5665/sleep.2810

81. Koh D, Meng KL, Sin EC, et al. Risk perception and impact of severe acute respiratory syndrome (SARS) on work and personal lives of healthcare workers in Singapore: What can we learn? Med Care. 2005. doi:10.1097/01.mlr.0000167181.36730.cc

82. Lancee WJ, Maunder RG, Goldbloom DS. Prevalence of psychiatric disorders among Toronto hospital workers one to two years after the SARS outbreak. Psychiatr Serv. 2008. doi:10.1176/ps.2008.59.1.91

83. Tam CWC, Pang EPF, Lam LCW, Chiu HFK. Severe acute respiratory syndrome (SARS) in Hongkong in 2003: Stress and psychological impact among frontline healthcare workers. Psychol Med. 2004. doi:10.1017/S0033291704002247

84. Hossain MM, Purohit N, Sultana A, Ma P, McKyer ELJ, Ahmed HU. Prevalence of mental disorders in South Asia: An umbrella review of systematic reviews and metaanalyses. Asian J Psychiatr. 2020. doi:10.1016/j.ajp.2020.102041

85. Zhang Y, Ren R, Lei F, et al. Worldwide and regional prevalence rates of co-occurrence of insomnia and insomnia symptoms with obstructive sleep apnea: A systematic review and meta-analysis. Sleep Med Rev. 2019. doi:10.1016/j.smrv.2019.01.004

86. Hossain MM. Current Status of Global Research on Novel Coronavirus Disease (COVID- 
19): A Bibliometric Analysis and Knowledge Mapping. SSRN. 2020.

https://ssrn.com/abstract=3547824 or http://dx.doi.org/10.2139/ssrn.3547824.

87. Zyoud SH, W. Al-Jabi S. Mapping the situation of research on coronavirus disease-19 (COVID-19): a preliminary bibliometric analysis during the early stage of the outbreak. BMC Infect Dis. 2020.

88. Lemke MK, Apostolopoulos Y, Sönmez S. Syndemic frameworks to understand the effects of COVID-19 on commercial driver stress, health, and safety. J Transp Heal. 2020. doi:10.1016/j.jth.2020.100877

89. Gravlee CC. Systemic racism, chronic health inequities, and COVID-19: A syndemic in the making? Am J Hum Biol. 2020. doi:10.1002/ajhb. 23482

90. Chandu VC, Marella Y, Panga GS, Pachava S, Vadapalli V. Measuring the Impact of COVID-19 on Mental Health: A Scoping Review of the Existing Scales. Indian J Psychol Med. 2020. doi:10.1177/0253717620946439

91. Hossain MM, Tasnim S, Sharma R, et al. Digital interventions for people living with noncommunicable diseases in India: A systematic review of intervention studies and recommendations for future research and development. Digit Heal. 2019. doi:10.1177/2055207619896153

92. Ramsetty A, Adams C. Impact of the digital divide in the age of COVID-19. J Am Med Inform Assoc. 2020. doi:10.1093/jamia/ocaa078

93. Torous J, Myrick KJ, Rauseo-Ricupero N, Firth J. Digital mental health and COVID-19: Using technology today to accelerate the curve on access and quality tomorrow. $J$ Med Internet Res. 2020. doi:10.2196/18848

94. Hood HK, Rogojanski J, Moss TG. Cognitive-Behavioral Therapy for Chronic Insomnia. Curr Treat Options Neurol. 2014. doi:10.1007/s11940-014-0321-6

95. A. VS, T. VDZ, C. M. Cognitive and behavioural therapies in the treatment of insomnia: A systematic metaanalysis of all the literature. J Sleep Res. 2018. doi:http://dx.doi.org/10.1111/jsr.12751 
96. Short NA, Schmidt NB. Developing and Testing a Novel, Computerized Insomnia and Anxiety Intervention to Reduce Safety Aids Among an at-Risk Student Sample: A Randomized Controlled Trial. Behav Ther. 2020. doi:10.1016/j.beth.2019.05.012

97. Zhou ES, Recklitis CJ. Internet-delivered insomnia intervention improves sleep and quality of life for adolescent and young adult cancer survivors. Pediatr Blood Cancer. 2020. doi:10.1002/pbc. 28506

98. Dekker K, Benjamins JS, Straten A Van, Hofman WF, Van Someren EJW. Effectiveness of internet-supported cognitive behavioral and chronobiological interventions and effect moderation by insomnia subtype: Study protocol of a randomized controlled trial. Trials. 2015. doi:10.1186/s13063-015-0790-2

99. Zhang X, Wang X, Le S, et al. Effects of exercise and dietary interventions on serum metabolites in men with insomnia symptoms: A 6-month randomized controlled trial. Sport Med Heal Sci. 2020. doi:10.1016/j.smhs.2020.05.002 